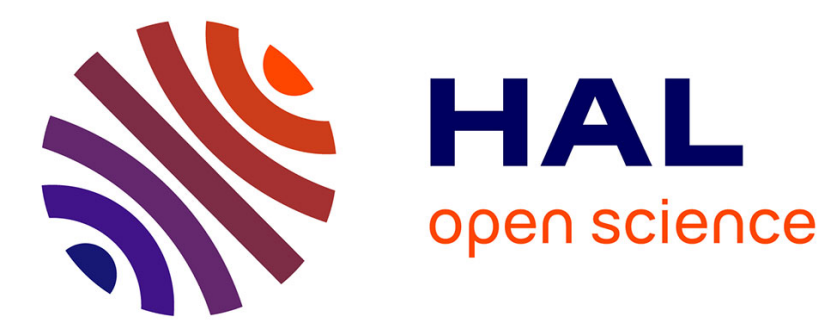

\title{
An approximate solution of the Riemann problem for a realisable second-moment turbulent closure
}

Christophe Berthon, Frédéric Coquel, Jean-Marc Hérard, Markus Uhlmann

\section{To cite this version:}

Christophe Berthon, Frédéric Coquel, Jean-Marc Hérard, Markus Uhlmann. An approximate solution of the Riemann problem for a realisable second-moment turbulent closure. Shock Waves, 2002, 11 (4), pp.245-269. 10.1007/s001930100109 . hal-01484338

\section{HAL Id: hal-01484338 \\ https://hal.science/hal-01484338}

Submitted on 10 Mar 2017

HAL is a multi-disciplinary open access archive for the deposit and dissemination of scientific research documents, whether they are published or not. The documents may come from teaching and research institutions in France or abroad, or from public or private research centers.
L'archive ouverte pluridisciplinaire HAL, est destinée au dépôt et à la diffusion de documents scientifiques de niveau recherche, publiés ou non, émanant des établissements d'enseignement et de recherche français ou étrangers, des laboratoires publics ou privés. 


\title{
An approximate solution of the Riemann problem for a realisable second-moment turbulent closure
}

\author{
C. Berthon ${ }^{1}$, F. Coquel $^{1}$, J.M. Hérard ${ }^{2,3}$, M. Uhlmann ${ }^{4}$
}

1 Université Paris VI, Laboratoire d'Analyse Numérique, 4 place Jussieu, 75008 Paris, France

2 Electricité de France, Direction des Etudes et Recherches, Département Laboratoire National d'Hydraulique, 6 quai Watier, 78400 Chatou, France

${ }^{3}$ Université de Provence, LATP-UMR 6632, Centre de Mathématique et d'Informatique, 39 rue Joliot Curie, 13453 Marseille cedex, France

${ }^{4}$ Ecole Centrale de Lyon, 36 avenue Guy de Collongue, 69131 Ecully, France

Received 2 March 1999 / Accepted 24 August 2000

\begin{abstract}
An approximate solution of the Riemann problem associated with a realisable and objective turbulent second-moment closure, which is valid for compressible flows, is examined. The main features of the continuous model are first recalled. An entropy inequality is exhibited, and the structure of waves associated with the non-conservative hyperbolic convective system is briefly described. Using a linear path to connect states through shocks, approximate jump conditions are derived, and the existence and uniqueness of the one-dimensional Riemann problem solution is then proven. This result enables to construct exact or approximate Riemann-type solvers. An approximate Riemann solver, which is based on Gallouët's recent proposal is eventually presented. Some computations of shock tube problems are then discussed.
\end{abstract}

Key words: Riemann problem, Approximate Riemann solver, Turbulent closure

\section{Nomenclature}

$f_{, i}$

$f_{, t}$

$\theta^{\prime \prime}=\theta-\langle\rho \theta\rangle /\langle\rho\rangle$
$\left\langle\theta^{\prime \prime}\right\rangle$

$\rho($ or $\langle\rho\rangle)$

$p($ or $\langle p\rangle)$

$T=\langle p\rangle /\langle\rho\rangle$

$E($ or $\langle E\rangle)$

$\eta$

$f_{\eta}^{\text {nv }}$

$f_{\eta}^{v}$

For $i=1 \rightarrow 3$ :

$U_{i}$ partial derivative of $f$ with respect to $x_{i}$ partial derivative of $f$ with respect to $t$

Reynolds average of instantaneous variable $\theta$

Favre decomposition of $\theta$

Reynolds average of fluctuation

(in the sense of Favre's averaging)

mean density

mean pressure

mean temperature

mean total energy

entropy

non-viscous part of entropy flux

viscous part of entropy flux dissipation rate of the turbulent kinetic

energy $K$

Correspondence to: J.M. Hérard

(e-mail: Herard@cmi.univ-mrs.fr)
For $i=1 \rightarrow 3$, and $j=1 \rightarrow 3$ :

$R_{i j}=\left\langle\rho u_{i}^{\prime \prime} u_{j}^{\prime \prime}\right\rangle \quad$ turbulent Reynolds stress tensor

$\Sigma_{i j}^{\mathrm{v}}=-\mu\left(U_{i, j}+U_{j, i}-\frac{2}{3} U_{1,1} \delta_{i j}\right)$

$\Phi_{i j} \quad \begin{aligned} & \text { molecular viscous strain tensor } \\ & \text { (so-called) pressure strain correlation }\end{aligned}$ tensor

For $i=1 \rightarrow 3$, and $j=1 \rightarrow 3$, and $k=1 \rightarrow 3$ :

$\Phi_{i j}^{k}=\left\langle\rho u_{i}^{\prime \prime} u_{j}^{\prime \prime} u_{k}^{\prime \prime}\right\rangle \quad$ triple velocity correlation

Reynolds stress tensor invariants:

$\mathrm{I}=2 K=$ trace $(\mathbf{R})$ turbulent kinetic energy

$\mathrm{II}_{\mathrm{R}}=\operatorname{trace}\left(\mathbf{R}^{2}\right) \quad$ second invariant of $\mathbf{R}$

$\mathrm{III}_{\mathrm{R}}=\operatorname{trace}\left(\mathbf{R}^{3}\right) \quad$ third invariant of $\mathbf{R}$

For $i=1 \rightarrow 3$ :

$\lambda_{i} \quad$ eigenvalues of the Reynolds stress

tensor $\mathbf{R}$

$\delta_{1}^{i}=R_{i i} \quad$ first fundamental minor of $\mathbf{R}$ (no

$\delta_{2}^{i}=R_{\alpha \alpha} R_{\beta \beta}-\left(R_{\alpha \beta}\right)^{2}$ summation) second fundamental minor of $\mathbf{R}$ (no summation); $\alpha$ and $\beta$ are not equal and not equal to $i$

$\delta_{3}=\operatorname{det}\left(R_{i j}\right) \quad$ third fundamental minor of $\mathbf{R}$ 


\section{Introduction}

We present herein some new results, which are expected to be useful for numerical studies of turbulent compressible flows using second order closures. These seem to be attractive from both theoretical and numerical points of view. Numerical investigation of turbulent compressible flows is usually carried out using one- or two-equation turbulent compressible models, such as the well-known $\mathrm{K}-\varepsilon$ closure, or computing the governing equations for all Reynolds stress components. Differential transport models are potentially capable of predicting the influence of a shock wave on the anisotropy of the Reynolds stress tensor, as idealized studies of shock wave/turbulence interaction suggest (Uhlmann 1997). Actually, both first order and second-moment models involve non-conservative first order differential systems. Two-equation turbulent closures have been recently investigated (Forestier et al. 1995, 1997; Hérard 1995b; Hérard et al. 1995; Louis 1995); these references suggest two different ways, namely, (i) a Godunov-type approach alike Godunov's (1959) original scheme (Forestier et al. 1997), and (ii) a flux-differencesplitting as originally proposed by Roe (extended to nonconservative systems, for instance, by Hérard 1995b), to compute convective fluxes and non-conservative terms, so that turbulent shock-tube problems might be numerically simulated. Both ways have been proven to be suitable tools for that purpose, and enable to preserve Riemann invariants in linearly degenerate fields. It was also confirmed numerically (Page and Uhlmann 1996) that standard Euler Riemann solvers are not adequate for that goal. Turning now to second-moment closures, preceeding remarks still hold; moreover, as underlined recently by Uhlmann (1997), Euler-type Riemann solvers can no longer be used to compute this kind of closures, since they provide unstable computational results in some situations, which are mainly due to the occurrence of two new waves, which do not exist in the Euler framework. Though emphasis is put on a simple objective and strongly realisable model arising from the literature, the present analysis extends to more complex closures proposed by Fu et al. (1987), Shih and Lumley (1985), Shih et al. (1994).

It must be emphasized here that a priori suitability of second-order compressible closures to predict the behaviour of flows including shocks (or even contact discontinuities) is beyond the purpose of the present paper, and might generate a huge discussion. Moreover, it is absolutely not claimed here that the approach is the most adequate one. In particular, one should at least distinguish the adequacy of second-moment closures in compressible flows including shock waves in terms of two distinct concepts. First of all, one may wonder whether continuous solutions satisfy some basic "intrinsic" concepts such as realisability (Lumley 1978), invariance under some frame rotation or translation (Speziale 1979), or some constraint pertaining to thermodynamics; this will be briefly discussed below, and many references to previous works will be recalled. A second way to evaluate these models is related to their ability to predict accurately unsteady complex situations encountered in well-documented experiments; as recalled above, many papers referenced in the work by Uhlmann (1997) address this problem. Thus, the basic two ideas underlined in the work are actually the following ones. Assuming the second-moment closure approach is indeed a suitable one, how should one try to compute unsteady flows including discontinuities (shocks and contact discontinuities) when applying this kind of closures to the averaged Navier-Stokes equations? And even more: may one exhibit analytical solutions of such a model, at least in a one-dimensional framework (though accounting for three spatial dimensions of the physical space)? The latter problem is indeed a delicate one since the occurrence of non-conservative terms in the first order set of partial differential equations renders the problem of formulating jump conditions much more difficult than in the purely conservative framework (see the basic works of Dal Maso et al. 1995; Le Floch 1988; Le Floch and Liu 1992 on that specific topic).

In the first three sections, governing equations and some basic properties of the whole viscous system are briefly described. Approximate jump conditions are then proposed, which are based on a linear path with respect to some non-conservative variable. This allows constructing the solution of the 1-D Riemann problem, applying for the entropy inequality, and restricting to weak shocks. The solution of the Riemann problem satisfies realisability requirements. These results enable us to propose in the fourth section a simple but efficient way to compute timedependent solutions including rarefaction waves, shocks and contact discontinuities. It should be emphasised here that other ways to provide approximate Riemann solvers based on strongly coupled upwinding techniques may be suggested, but the main purpose of the present contribution is not to exhibit the "ultimate" scheme but to take advantage of the continuous analysis to exhibit an expected meaningful compromise between efficiency and accuracy. In particular, the comparison with commonly used Roe's method may be found in the work by Uhlmann (1997), though the latter scheme does not comply exactly with so-called Roe's condition (which corresponds to the consistency with the integral form of the conservation law, when focusing on conservation laws). The fifth section will thus be devoted to the presentation of sample computational results of turbulent shock tube problems, which confirm the capabilities of the scheme, even when the turbulent Mach number is close to one.

\section{Set of equations}

Before we examine the solution of the one-dimensional Riemann problem associated with the whole governing set of equations, and then introduce the approximate Riemann solver which is used to compute turbulent compressible flows with shocks, we first recall some basic results connected with a simple strongly-realisable secondmoment closure, which is based on standard Favre's (1965) averaging. All commonly used averaging symbols (overtilda for the Favre averaging) have been dropped herein to avoid confusion between instantaneous and averaged 
values of functions. Brackets \langle\rangle$-$ which are present in some places - refer to the Reynolds averaging of variables, instead of standard overbar notations. The latter will be used for the arithmetic average when discussing numerical implementation in finite-volume codes. The nomenclature is intended to help readers who are not very familiar with these notations. Hence, the set of equations is (see the work by Vandromme and Ha Minh (1986) for a general presentation):

$$
\begin{gathered}
(\rho)_{, t}+\left(\rho U_{i}\right)_{, i}=0 \\
\left(\rho U_{i}\right)_{, t}+\left(\rho U_{i} U_{j}\right)_{, j}+\left(p \delta_{i j}\right)_{, j}+\left(R_{i j}\right)_{, j}=-\left(\Sigma_{i j}^{\mathrm{v}}\right)_{, j} \\
(E)_{, t}+\left(E U_{j}\right)_{, j}+\left(U_{i}\left(p \delta_{i j}+R_{i j}\right)\right)_{, j}=-\left(U_{i}\left(\Sigma_{i j}^{\mathrm{v}}\right)\right)_{, j} \\
+\left(\sigma_{\mathrm{E}}\left(\frac{p}{\rho}\right)_{j}\right)_{, j}+\left(\frac{\Phi_{j j}^{k}}{2}\right)_{, k}-\left(\left\langle u_{i}^{\prime \prime}\right\rangle p\right)_{, i} ; \\
\left(R_{i j}\right)_{, t}+\left(R_{i j} U_{k}\right)_{, k}+R_{i k} U_{j, k}+R_{j k} U_{i, k}=-\left\langle u_{i}^{\prime \prime}\right\rangle p_{, j} \\
-\left\langle u_{j}^{\prime \prime}\right\rangle p_{, i}+\Phi_{i j}-\frac{2}{3}\left(\frac{\varepsilon}{I}\right) \operatorname{trace}(\mathbf{R}) \delta_{i j}+\left(\Phi_{i j}^{k}\right)_{, k},
\end{gathered}
$$

with

$$
\begin{gathered}
R_{i j}=\left\langle\rho u_{i}^{\prime \prime} u_{j}^{\prime \prime}\right\rangle \\
\Sigma_{i j}^{\mathrm{v}=-\mu}\left(U_{i, j}+U_{j, i}-\frac{2}{3} U_{1,1} \delta_{i j}\right) .
\end{gathered}
$$

Moreover, it is assumed that the perfect gas state law holds. Hence:

$$
p=(\gamma-1)\left(E-\frac{\rho U_{j} U_{j}}{2}-\frac{1}{2} R_{j j}\right) .
$$

Given some (normalised) vector $\mathbf{n}$ in $I R^{3}$, admissible states should comply with the over-realisability constraints

$$
R_{n n}(\mathbf{x}, t)=\mathbf{n}^{t} \mathbf{R}(\mathbf{x}, t) \mathbf{n}>0
$$

and be such that

$$
\begin{aligned}
& \rho(\mathbf{x}, t) \geq 0 ; \\
& p(\mathbf{x}, t) \geq 0 .
\end{aligned}
$$

We also need to introduce:

$$
U_{n}(\mathbf{x}, t)=\mathbf{U}^{t}(\mathbf{x}, t) \mathbf{n} .
$$

Above, $\rho$ stands for the mean density, $\mathbf{U}$ is the mean velocity, $E$ denotes the mean total energy and $p$ is the mean pressure. The Reynolds stress tensor is $\mathbf{R}$, and $K$ is the turbulent kinetic energy $(K=$ trace $(\mathbf{R}) / 2) ; \gamma$ (greater than one) is the ratio of specific heats and $\sigma_{E}$ is a positive function; $\mu$ is the molecular viscosity. A standard equation governs the motion of the mechanical dissipation $\varepsilon$. The turbulent mass flux is modelled according to the proposition of Ristorcelli (1993):

$$
\left\langle u_{i}^{\prime \prime}\right\rangle=\tau \frac{R_{i j}}{\rho^{2}} \rho_{, j},
$$

where $\tau$ represents some turbulent time scale. Basic constraints are recalled now. First of all, solutions of the whole closed set should be such that $(8 \mathrm{a}-\mathrm{c})$ hold. In particular, (8a) gives:

$$
\begin{gathered}
R_{i i} \geq 0 \quad(i=1,2,3) \\
R_{i i} R_{j j}-R_{i j}^{2} \geq 0 \quad(1 \leq i \leq j \leq 3) \\
R_{11} R_{22} R_{33}+2 R_{12} R_{13} R_{23}-R_{33} R_{12}^{2}-R_{11} R_{23}^{2} \\
-R_{22} R_{13}^{2} \geq 0 .
\end{gathered}
$$

Any positive quantity $\theta$ (chosen among the fundamental minors of the Reynolds stress tensor $\mathbf{R}$ ) should behave as follows when vanishing (see $\mathrm{Fu}$ et al. 1987; Hérard 1994, 1995a, 1996; Lumley 1978, 1983; Pope 1985; Shih and Lumley 1985; Shih et al. 1993):

$$
\theta_{M}=0 \Rightarrow\left\{\left(D_{t} \theta\right)_{M}=0 \text { and }\left(D_{t}\left(D_{t} \theta\right)\right)_{M} \geq 0\right\} .
$$

Lumley (1978) proposed a simple model for the pressurestrain term $\Phi_{i j}$, which is in agreement with the previous constraints. We thus focus on this closure, which accounts for the return-to-isotropy process and fulfils the objectivity requirement (Hérard 1994; Lumley 1978; Speziale 1979). Hence:

$$
\Phi_{i j}=-\frac{\varepsilon}{I}\left\{\left(2+f \frac{\delta_{3}}{I^{3}}\right)\left(R_{i j}-\frac{I \delta_{i j}}{3}\right)\right\},
$$

where $\delta_{3}$ denotes the product of the three eigenvalues of the Reynolds stress tensor, i.e.

$$
\delta_{3}=\lambda_{1} \lambda_{2} \lambda_{3}
$$

and $I$ stands for the trace of the Reynolds stress tensor:

$$
I=R_{i i} .
$$

The exact form of the function $f$ is given in the original paper by Lumley (1978).

Finally, it is assumed that the turbulent velocity field almost follows a Gaussian distribution, and we thus neglect the triple velocity correlation:

$$
\Phi_{i j}^{k}=0 .
$$

\section{Some basic results}

\subsection{Entropy inequality}

We introduce the so-called "conservative" state variable:

$$
\mathbf{W}^{t}=\left(\rho, \rho U, \rho V, \rho W, E, R_{11}, R_{22}, R_{33}, R_{12}, R_{13}, R_{23}\right) .
$$

Focusing on regular solutions of set (1-4), the following may be easily derived:

Proposition 1.

Define:

$$
\eta(\mathbf{W})=-\rho \log \left(p \rho^{-\gamma}\right)
$$


and

$$
f_{\eta}^{\mathrm{nv}}(\mathbf{W})=\mathbf{U} \eta
$$

Regular solutions $W$ of set (1-4) are such that:

$\eta_{, t}+\nabla \cdot\left(f_{\eta}^{\mathrm{nv}}(\mathbf{W})\right)+\nabla \cdot\left(f_{\eta}^{\mathrm{v}}(\mathbf{W}, \nabla \mathbf{W})\right)=S_{\eta}(\mathbf{W}, \nabla \mathbf{W}) \leq 0$.

The source term may be written as:

$$
\begin{aligned}
S_{\eta}(\mathbf{W}, \nabla \mathbf{W})= & -\frac{\gamma-1}{T} \\
& \times\left(\frac{\sigma_{\mathrm{E}}}{T} T_{, i} T_{, i}+\varepsilon-\frac{1}{2} \Sigma_{i j}^{\mathrm{v}}\left(U_{i, j}+U_{j, i}\right)\right) \\
& -(\gamma-1) \frac{\tau}{\rho^{2}} \rho_{, i} R_{i j} \rho_{, j}
\end{aligned}
$$

(noting that $T=p / \rho$ ). The viscous flux is such that $f_{\eta}^{\mathrm{v}}(\mathbf{W}, \nabla \mathbf{W})=0$ if $\nabla \mathbf{W}=0$. Thus, the entropy inequality reduces to

$$
-\sigma[\eta]+\left[f_{\eta}^{\mathrm{nv}}\right] \leq 0
$$

in the non-viscous limit. It will be helpful to connect states through genuinely non-linear fields in a physical way, when neglecting viscous effects. This entropy inequality is similar to one arising when investigating one- and two-equation models (Forestier et al. 1997; Hérard 1995b).

\subsection{A conservative entropy-consistent splitting-up technique}

We now introduce the following splitting-up technique, which requires solving a first-order differential system including a very small amount of laminar viscous effects, and a second step which treats both source terms and second order terms. The main advantage is that this technique enables including mass flux contributions (as described above), if needed. Another interesting point is that it isolates terms arising from turbulence modelling in step II, whereas step I only involves non-controversial contributions. Hence, we set:

\section{Step I:}

$$
\begin{aligned}
& (\rho)_{, t}+\left(\rho U_{i}\right)_{, i}=0 \\
& \left(\rho U_{i}\right)_{, t}+\left(\rho U_{i} U_{j}\right)_{, j}+\left(p \delta_{i j}\right)_{, j}+\left(R_{i j}\right)_{, j}=-\theta\left(\Sigma_{i j}^{\mathrm{v}}\right)_{, j} \\
& (E)_{, t}+\left(E U_{j}\right)_{, j}+\left(U_{i}\left(p \delta_{i j}+R_{i j}\right)\right)_{, j}=-\theta\left(U_{i}\left(\Sigma_{i j}^{\mathrm{v}}\right)\right)_{, j} \\
& \left(R_{i j}\right)_{, t}+\left(R_{i j} U_{k}\right)_{, k}+R_{i k} U_{j, k}+R_{j k} U_{i, k}=0 .
\end{aligned}
$$

\section{Step II:}

$$
\begin{aligned}
(\rho)_{, t}=0 & \\
\left(\rho U_{i}\right)_{, t}= & -(1-\theta)\left(\Sigma_{i j}^{\mathrm{v}}\right)_{, j} ; \\
(E)_{, t}= & -(1-\theta)\left(U_{i}\left(\Sigma_{i j}^{\mathrm{v}}\right)\right)_{, j}+\left(\sigma_{\mathrm{E}}\left(\frac{p}{\rho}\right)_{j}\right)_{, j} \\
& -\left(\left\langle u_{i}^{\prime \prime}\right\rangle p\right)_{, i} ; \\
\left(R_{i j}\right)_{, t}= & -\left\langle u_{i}^{\prime \prime}\right\rangle p_{, j}-\left\langle u_{j}^{\prime \prime}\right\rangle p_{, i}+\Phi_{i j}-\frac{2}{3}\left(\frac{\varepsilon}{I}\right) \operatorname{trace}(\mathbf{R}) \delta_{i j} .
\end{aligned}
$$

The parameter $\theta$ is assumed to lie in [0,1]. Numerical simulations of the system involved in step I will be depicted later setting $\theta$ to zero. Note that all second order terms in step II are in conservative form, unless turbulent mass fluxes are accounted for. Regular enough solutions of step I agree with

$$
\eta_{, t}+\left(U_{j} \eta\right)_{, j}=-\theta \frac{\gamma-1}{T}\left(-\frac{1}{2} \Sigma_{i j}^{\mathrm{v}}\left(U_{i, j}+U_{j, i}\right)\right) \leq 0,
$$

the non-viscous limit of which being

$$
\eta_{, t}+\left(U_{j} \eta\right)_{, j} \leq 0 .
$$

In a similar way, smooth solutions of step II satisfy

$$
\begin{aligned}
\eta_{, t}+ & \left(\left(f_{\eta}^{\mathrm{v}}\right)_{j}\right)_{, j} \\
=- & \frac{\gamma-1}{T}\left(\frac{\sigma_{\mathrm{E}}}{T} T_{, i} T_{, i}+\varepsilon-\frac{1-\theta}{2} \Sigma_{i j}^{\mathrm{v}}\left(U_{i, j}+U_{j, i}\right)\right) \\
& \quad-(\gamma-1)\left\langle u_{j}^{\prime \prime}\right\rangle \rho_{, j} .
\end{aligned}
$$

This results in

$$
\eta, t \leq 0
$$

in the non-viscous limit, whenever turbulent mass fluxes are neglected or chosen to be in agreement with the former proposition: $\left\langle u_{i}^{\prime \prime}\right\rangle=\tau\left(R_{i j} / \rho^{2}\right) \rho_{, j}$. This approach may be extended to the framework of some more complex closures where so-called rapid pressure-strain correlations are accounted for in a suitable way. This is not discussed herein.

From now on, we focus on the most difficult part, namely step I.

\subsection{Hyperbolicity criteria for the non-conservative convection system}

We focus now on statistically two-dimensional turbulence, and hence assume that

$$
R_{13}=R_{23}=0, \quad W=0
$$

and also

$$
\varphi_{, 3}=0
$$

whatever $\varphi$ stands for. We also introduce a " $2 \mathrm{D}$ conservative" state variable:

$$
\mathbf{Z}^{t}=\left(\rho, \rho U, \rho V, E, R_{11}, R_{22}, R_{12}, R_{33}\right) .
$$

The non-conservative convective subset arising from (1-4) (or step I) reads:

$$
\mathbf{Z}_{, t}+\sum_{i=1}^{2}\left(\mathbf{F}_{i}(\mathbf{Z})\right)_{, i}+\sum_{i=1}^{2} \mathbf{A}_{i}^{\mathrm{nc}}(\mathbf{Z}) \mathbf{Z}_{, i}=0 .
$$

This enables to derive

\section{Proposition 2.}

Define:

$$
c_{1}=\left(\frac{\gamma p}{\rho}+3 \frac{R_{n n}}{\rho}\right)^{1 / 2}
$$




$$
c_{2}=\left(\frac{R_{n n}}{\rho}\right)^{1 / 2} .
$$

(i) The two-dimensional non-conservative first order partial differential set (10) is a non-strictly hyperbolic system if $(8 a-8 c)$ holds. Eigenvalues are:

$$
\begin{aligned}
\lambda_{1} & =U_{n}-c_{1} ; \\
\lambda_{2} & =U_{n}-c_{2} ; \\
\lambda_{3}=\lambda_{4} & =\lambda_{5}=\lambda_{6}=U_{n} ; \\
\lambda_{7} & =U_{n}+c_{2} ; \\
\lambda_{8} & =U_{n}+c_{1} .
\end{aligned}
$$

(ii) In a one-dimensional framework, the 1-wave and the 8-wave are genuinely non-linear; other fields are linearly degenerate.

The computation of eigenvalues and right eigenvectors is tedious but straightforward. We refer to the books by Godlewski and Raviart (1996), Smoller (1983) for basic concepts and definitions to investigate hyperbolic systems under conservation form. It seems worth noting the occurrence of two new contact discontinuities with respect to the Euler framework. It must be noted that the eigenvalue problem is "ill-conditioned", due to the fact that the turbulent Mach number, which is proportional to $(K / p)^{1 / 2}$ is usually much smaller than one. A sketch of waves is given in Fig. 1.

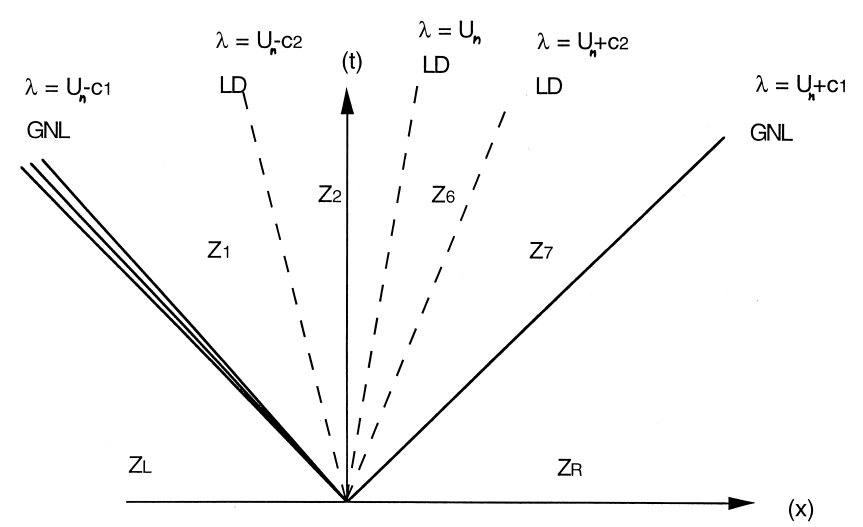

Fig. 1. Sketch of waves: GNL - genuinely non-linear wave; LD - linearly degenerate wave

Let us note also that, due to the fact that in most practical applications the turbulent intensity $K$, which varies as $R_{n n}$, is small as compared with either the sound propagation in the laminar case or even with the mean velocity $U$ in the turbulent case, intermediate states $\mathbf{Z}_{2}$ and $\mathbf{Z}_{6}$ will be difficult to distinguish from each other; this will be confirmed by numerical experiments. This remark still holds in the multi-dimensional case, whenever one uses structured or unstructured meshes; however, the direction of the outward normal vector to a cell interface may modify (increase or decrease) the value of the ratio $\left(U \cdot n / R_{n n}\right)$ at a given position in space.

\section{An approximate solution of the one-dimensional Riemann problem}

From now on we restrict ourselves to shocks of small amplitude. We afterwards connect states $\left(\rho^{-1}, U, V, p, R_{11}\right.$, $R_{22}, R_{33}, R_{12}$ ) with a linear path (see Dal Maso et al. 1995; Le Floch 1988; Le Floch and Liu 1992; Sainsaulieu 1995a,b) across discontinuities. If $\sigma$ stands for the speed of a travelling discontinuity, the following approximate jump conditions arise (we set $[\varphi]_{l}^{r}=\varphi_{r}-\varphi_{l}$ and $\bar{\varphi}_{l r}=$ $\left.\left(\varphi_{r}+\varphi_{l}\right) / 2\right)$ :

$$
\begin{gathered}
-\sigma[\rho]_{l}^{r}+[\rho U]_{l}^{r}=0 ; \\
-\sigma[\rho U]_{l}^{r}+\left[\rho U^{2}+R_{11}+p\right]_{l}^{r}=0 ; \\
-\sigma[\rho V]_{l}^{r}+\left[\rho U V+R_{12}\right]_{l}^{r}=0 ; \\
-\sigma[E]_{l}^{r}+\left[U\left(E+R_{11}+p\right]_{l}^{r}+\left[V R_{12}\right]_{l}^{r}=0 ;\right. \\
-\sigma\left[R_{11}\right]_{l}^{r}+\left[U R_{11}\right]_{l}^{r}+2 \overline{\left(R_{11}\right)_{l r}}[U]_{l}^{r}=0 ; \\
-\sigma\left[R_{22}\right]_{l}^{r}+\left[U R_{22}\right]_{l}^{r}+2 \overline{\left(R_{12}\right)_{l r}}[V]_{l}^{r}=0 ; \\
-\sigma\left[R_{12}\right]_{l}^{r}+\left[U R_{12}\right]_{l}^{r}+\overline{\left(R_{11}\right)_{l r}}[V]_{l}^{r}+\overline{\left(R_{12}\right)_{l r}}[U]_{l}^{r}=0 ; \\
-\sigma\left[R_{33}\right]_{l}^{r}+\left[U R_{33}\right]_{l}^{r}=0 .
\end{gathered}
$$

Obviously, these approximate jump conditions turn out to be the exact ones when the turbulence vanishes (i.e. setting $R_{11}=R_{22}=R_{33}=R_{12}=0$ ). Owing to the entropy inequality

$$
-\sigma[\eta]+\left[f_{\eta}^{\mathrm{nv}}\right] \leq 0
$$

it may be proven that the 1D Riemann problem with sufficiently weak shocks has a unique entropy-consistent solution.

\section{Proposition 3.}

Define:

$$
\begin{aligned}
X_{i}= & \left(\frac{\gamma p_{i}}{\rho_{i}}\right)^{1 / 2} \\
& \times \int_{0}^{\rho_{i}}\left(\frac{a}{\rho_{i}}\right)^{(\gamma-1) / 2}\left(1+3 \frac{\left(R_{n n}\right)_{i}}{\gamma p_{i}}\left(\frac{a}{\rho_{i}}\right)^{3-\gamma}\right)^{1 / 2} \frac{\mathrm{d} a}{a}
\end{aligned}
$$

for $i=L, R$. Assume that initial data is in agreement with $(8 a-c)$. Then, the one-dimensional Riemann problem associated with the non-conservative system (10), abovementioned approximate jump conditions, and initial data

$$
\begin{aligned}
& \mathbf{Z}(x<0, t=0)=\mathbf{Z}_{\mathbf{L}}, \\
& \mathbf{Z}(x>0, t=0)=\mathbf{Z}_{\mathbf{R}}
\end{aligned}
$$

has a unique solution in agreement with conditions (8a) if the following condition holds:

$$
\left(U_{n}\right)_{R}-\left(U_{n}\right)_{L}<X_{L}+X_{R}
$$

The solution is such that the mean pressure and the mean density remain positive in the $(x, t)$ plane.

The reader is referred to Appendices A1-A4 for proof. 
Remark 1. The above-mentioned condition of existence and uniqueness is the counterpart of the well-known condition of vacuum occurrence when investigating the onedimensional Riemann problem in gas dynamics (Smoller 1983) and restricting to the perfect gas state laws. It must be emphasized that the realisability of the Reynolds stress tensor is preserved, through contact discontinuities, but also through genuinely non-linear fields, whenever these turn out to be shocks or rarefaction waves (see Appendix $\mathrm{A} 5)$.

Remark 2. It is clear that the solution of the one-dimensional Riemann problem is close to that associated with the Riemann problem arising in one- or two-equation turbulent models (Forestier et al. 1997). In the present case, the leading variables are, in fact, $\rho, U_{n}, p, R_{n n}$; this means that for given initial data for $\rho, U_{n}, p, R_{n n}$ on both sides of the initial interface, the solution is completely determined, independently of the values for the remaining components. This is due to the fact that the above quantities remain constant through the two new contact discontinuities, since we get, using notations introduced in Fig. 1:

$$
\begin{aligned}
& {[\rho]_{1}^{2}=\left[U_{n}\right]_{1}^{2}=[p]_{1}^{2}=\left[R_{n n}\right]_{1}^{2}=0 ;} \\
& {[\rho]_{6}^{7}=\left[U_{n}\right]_{6}^{7}=[p]_{6}^{7}=\left[R_{n n}\right]_{6}^{7}=0 .}
\end{aligned}
$$

\section{An approximate Riemann solver}

We focus here on the computation of step I using $\theta=0$. The computation of viscous fluxes, which are present in the right hand side of equations (2) and (3), requires only the implementation of central schemes. Moreover, suitable ways to implement source terms in step II (or, equivalently, the so-called "slow terms" in the right hand side of equation (4)) were discussed in a previous work (Hérard 1995a).

\subsection{Introductory remarks}

We obviously restrict ourselves here to finite-volume techniques (Eymard et al. 2001) due to the great complexity of the whole non-linear system. On the basis of the abovementioned result, a classical Godunov scheme - in the limit of weak shocks - based upon the linear path, may be developed to compute convective effects (Forestier et al. 1997); this, however, requires a tremendous amount of CPU time. Thus, if we intend to avoid complexity, the only schemes of practical interest seem to be approximate Riemann solvers. Since no exact Roe-type Riemann solver (Roe 1981) based on a state average may be exhibited in the present case (Uhlmann 1997), we present now an extension of the approximate Riemann solver called VFRoe which was recently proposed in order to deal with complex hyperbolic systems (Gallouët and Masella 1996; Masella et al. 1999). Actually, other extensions of standard schemes to the framework of non-conservative systems might be used for computational purposes, but we nonetheless restrict our presentation to VFRoe with nonconservative variables. It seems clear that either the HLL scheme (Harten et al. 1983), or its extension such as the HLLC scheme (Toro 1997) in order to account for the three distinct contact discontinuities, are also natural candidates for that purpose. Sample results with the help of the Rusanov scheme and an approximate form of the Roe scheme will be very briefly discussed as well.

In order to simplify the presentation, we now consider statistically one-dimensional flows in the $x$-direction, and thus define a "conservative" vector state $\mathbf{Z}^{t}=(\rho, \rho U, \rho V, E$, $\left.R_{11}, R_{22}, R_{12}, R_{33}\right)$, and introduce a matrix $\mathbf{B}_{1}(\mathbf{Z})$ :

$$
\mathbf{B}_{1}(\mathbf{Z})=\frac{\mathrm{d} \mathbf{F}_{1}}{\mathrm{~d} \mathbf{Z}}(\mathbf{Z})+\mathbf{A}_{1}^{\mathrm{nc}}(\mathbf{Z}) .
$$

We recall that

$$
\mathbf{F}_{1}(\mathbf{Z})=\left(\begin{array}{c}
\rho U \\
\rho U^{2}+p+R_{11} \\
\rho U V+R_{12} \\
U\left(E+p+R_{11}\right)+V R_{12} \\
U R_{11} \\
U R_{22} \\
U R_{12} \\
U R_{33}
\end{array}\right) .
$$

We introduce a constant time step $\Delta t$, integrate Eq. (10) over cell $\Omega_{i}$, which provides:

$$
\begin{gathered}
\left\{\int_{\Omega_{i}} \mathbf{Z}\left(x, t^{n+1}\right) \mathrm{d} x-\int_{\Omega_{i}} \mathbf{Z}\left(x, t^{n}\right) \mathrm{d} x\right\} \\
+\int_{t^{n}}^{t^{n+1}} \int_{\Omega_{i}}\left\{\left(\mathbf{F}_{1}(\mathbf{Z}(x, t))\right)_{, x}+\mathbf{A}_{1}^{\mathrm{nc}}(\mathbf{Z}(x, t)) \mathbf{Z}_{, x}\right\} \mathrm{d} t \mathrm{~d} x=0 .
\end{gathered}
$$

The explicit (first-order) numerical scheme is:

$$
\begin{aligned}
& \operatorname{vol}\left(\Omega_{i}\right)\left(\mathbf{Z}_{i}^{n+1}-\mathbf{Z}_{i}^{n}\right) \\
& \quad+\Delta t \int_{\Gamma_{i}} \mathbf{F}_{1}^{\text {num }}\left(\mathbf{Z}^{n}\right) \mathrm{d} \Gamma+\Delta t \mathbf{S}_{i}\left(\mathbf{Z}^{n}\right)=0,
\end{aligned}
$$

where:

$$
\begin{aligned}
& \mathbf{S}_{i}\left(\mathbf{Z}^{n}\right) \\
& =\left(\begin{array}{c}
0 \\
0 \\
0 \\
0 \\
2\left(\widehat{R_{11}}\right)_{i}\left(\hat{U}_{i+1 / 2}-\hat{U}_{i-1 / 2}\right) \\
2\left(\widehat{R_{12}}\right)_{i}\left(\hat{V}_{i+1 / 2}-\hat{V}_{i-1 / 2}\right) \\
\left(\widehat{R_{11}}\right)_{i}\left(\hat{V}_{i+1 / 2}-\hat{V}_{i-1 / 2}\right)+\left(\widehat{R_{12}}\right)_{i}\left(\hat{U}_{i+1 / 2}-\hat{U}_{i-1 / 2}\right) \\
0
\end{array}\right)
\end{aligned}
$$

The most suitable way to compute the so-called "source" terms, which is based on the recent proposals by Forestier et al. (1997) and Masella (1997), is described below. We now present the main features of the approximate Riemann solver. 


\subsection{A simple Riemann solver}

Though the scheme presented below is slightly different from the original VFRoe scheme (see Gallouët and Masella 1996), it has been shown that it provides similar rates of convergence, when focusing on the isentropic or full Euler set of equations. On the basis of a series of analytical test cases involving shocks, contact discontinuities and rarefaction waves, it was checked in this framework that the present VFRoe scheme is as accurate as Roe's scheme. This new approach has previously been used to compute some two-phase flows (in this case, no Roe-type approximate Riemann solver can be constructed, see the work of Declercq-Xeuxet 1999 and Faucher 2000), using finite-volume techniques.

First, using (12), Eq. (10) may be rewritten as follows:

$$
\mathbf{Z}(\mathbf{Y})_{, t}+\mathbf{B}_{1}(\mathbf{Z}(\mathbf{Y}))(\mathbf{Z}(\mathbf{Y}))_{, x}=0
$$

(restricting to regular solutions) where

$$
\mathbf{Y}^{t}=\left(\rho^{-1}, U, V, p, R_{11}, R_{22}, R_{12}, R_{33}\right) .
$$

Thus we have

$$
(\mathbf{Y})_{, t}+\mathbf{Z}_{Y}^{-1}(\mathbf{Y})(\mathbf{B})_{1}(\mathbf{Z}(\mathbf{Y})) \mathbf{Z}_{\mathbf{Y}}(\mathbf{Y})(\mathbf{Y})_{, x}=0
$$

or equivalently:

$$
(\mathbf{Y})_{, t}+\mathbf{C}_{1}(\mathbf{Y})(\mathbf{Y})_{, x}=0 .
$$

The 1D Riemann problem associated with the non-linear hyperbolic system (16) and initial conditions

$$
\begin{aligned}
& \mathbf{y}(x<0, t=0) \equiv \mathbf{Y}_{L}=\mathbf{Y}\left(\mathbf{Z}_{L}\right) \\
& \mathbf{y}(x>0, t=0) \equiv \mathbf{Y}_{R}=\mathbf{Y}\left(\mathbf{Z}_{R}\right)
\end{aligned}
$$

is linearized as follows:

$$
(\mathbf{Y})_{, t}+\mathbf{C}_{1}(\overline{\mathbf{Y}})(\mathbf{Y})_{, x}=0,
$$

where $\overline{\mathbf{Y}}=\left(\mathbf{Y}_{R}+\mathbf{Y}_{L}\right) / 2$. System (17) contains five distinct linearly degenerate fields, and its solution is trivial, since it only requires computing eight real coefficients noted $\alpha_{i}$ (for $i=1$ to 8 ), setting

$$
\mathbf{Y}_{R}-\mathbf{Y}_{L}=\sum_{i=1}^{8} \alpha_{i} \hat{\mathbf{r}}_{i}
$$

where $\left(\hat{\mathbf{r}}_{i}\right)$ represents the basis of right eigenvectors of the matrix $\mathbf{C}_{1}(\overline{\mathbf{Y}})$. Intermediate states are then uniquely defined (see Appendix B for details) as:

$$
\begin{gathered}
\mathbf{Y}_{1}=\mathbf{Y}_{L}+\alpha_{1} \hat{\mathbf{r}}_{1} ; \\
\mathbf{Y}_{2}=\mathbf{Y}_{L}+\alpha_{1} \hat{\mathbf{r}}_{1}+\alpha_{2} \hat{\mathbf{r}}_{2} ; \\
\mathbf{Y}_{6}=\mathbf{Y}_{R}-\alpha_{8} \hat{\mathbf{r}}_{8}-\alpha_{7} \hat{\mathbf{r}}_{7} ; \\
\mathbf{Y}_{7}=\mathbf{Y}_{R}-\alpha_{8} \hat{\mathbf{r}}_{8} ;
\end{gathered}
$$

and hence the state $\mathbf{Y}^{*}$ at the initial location of the data discontinuity is given by

$$
\begin{gathered}
\mathbf{Y}^{*}=\mathbf{Y}_{L} \text { if } \widehat{\lambda_{1}}>0 ; \\
\mathbf{Y}^{*}=\mathbf{Y}_{1} \text { if } \widehat{\lambda_{1}}<0 \text { and } \widehat{\lambda_{2}}>0 ; \\
\mathbf{Y}^{*}=\mathbf{Y}_{2} \text { if } \widehat{\lambda_{2}}<0 \text { and } \widehat{\lambda_{3}}>0 ; \\
\mathbf{Y}^{*}=\mathbf{Y}_{6} \text { if } \widehat{\lambda_{6}}<0 \text { and } \widehat{\lambda_{7}}>0 ; \\
\mathbf{Y}^{*}=\mathbf{Y}_{7} \text { if } \widehat{\lambda_{7}}<0 \text { and } \widehat{\lambda_{8}}>0 ; \\
\mathbf{Y}^{*}=\mathbf{Y}_{R} \text { if } \widehat{\lambda_{8}}<0,
\end{gathered}
$$

where

$$
\begin{gathered}
\widehat{\lambda_{1}}=\bar{U}-\widehat{c_{1}} ; \quad \widehat{\lambda_{2}}=\bar{U}-\widehat{c_{2}} ; \\
\widehat{\lambda_{3}}=\widehat{\lambda_{4}}=\widehat{\lambda_{5}}=\widehat{\lambda_{6}}=\bar{U} ; \\
\widehat{\lambda_{7}}=\bar{U}+\widehat{c_{2}} ; \quad \widehat{\lambda_{8}}=\bar{U}+\widehat{c_{1}} ;
\end{gathered}
$$

and also

$$
\begin{gathered}
\widehat{c_{1}}=\left(\bar{\tau}\left(\gamma \bar{p}+3 \overline{R_{11}}\right)\right)^{1 / 2} ; \\
\widehat{c_{2}}=\left(\bar{\tau} \overline{R_{11}}\right)^{1 / 2} .
\end{gathered}
$$

The numerical flux in the first integral on the left hand side of (14) is then set as:

$$
\mathbf{F}_{1}^{\text {num }}\left(\mathbf{Z}^{n}\right)=\mathbf{F}_{1}\left(\mathbf{Z}\left(\mathbf{Y}^{*}\right)\right) .
$$

The most obvious way to compute integrals in (15) is to apply central schemes:

$$
\hat{\phi}_{i}=\phi_{i}^{n} \quad \text { and } \quad \hat{\phi}_{i+1 / 2}=\left(\phi_{i}^{n}+\phi_{i+1}^{n}\right) / 2 .
$$

This approach is suitable when using Roe-type schemes (modified to account for non-conservative terms, Hérard 1995a,b). However, the most stable scheme when applied to Godunov-type schemes is obtained via

$$
\hat{\phi}_{i}=\frac{\phi_{i-1 / 2}^{*}+\phi_{i+1 / 2}^{*}}{2} \quad \text { and } \quad \hat{\phi}_{i+1 / 2}=\phi_{i+1 / 2}^{*}
$$

(to our knowledge, first proposed by Masella 1997).

Remark 3. The latter form (Masella 1997) to account for non-conservative terms is the straightforward counterpart of the Godunov scheme introduced previously to compute two-equation turbulent compressible closures (Louis 1995).

Remark 4. It should be emphasized that the present VFRoe scheme turns out to be the original Godunov scheme when considering the scalar Burgers equation rewritten in nonconservative

form, provided that the "star" value is the exact solution of the Riemann problem on the initial interface $\xi=$ $x / t=0$.

Remark 5. Obviously, an entropy correction is required (Buffard et al. 1998, 1999, 2000; Gallouët and Masella 1996; Masella 1997; Masella et al. 1999) to compute shock tube problems when one sonic point is present in the 1rarefaction wave (respectively, in the 8-rarefaction wave).

Remark 6 . An interesting property of the approximate Riemann solver may be exhibited (see Buffard et al. 2000 for a straightforward counterpart in the Euler framework): when considering a single discontinuity, it occurs that the jump conditions associated with (17) are equivalent to those detailed in Sect. 4. 


\section{Turbulent shock tube problems}

Shock tube problems are of prime importance to validate the numerical treatment of convective terms. Before we examine the capabilities of the scheme to predict the behaviour of turbulent shock tube flows, we briefly depict its speed of convergence when applied to the simulation of the Euler equations with the perfect gas state law (the ratio of specific heats $\gamma$ is assumed to be equal to $7 / 5$ ). When dealing with the Euler equations, the results obtained with the present scheme are in excellent agreement with those reported by Gallouët and Masella (1996), Masella (1997), Masella et al. (1999) and were also compared with those obtained using Roe's scheme or the Godunov scheme. Actually (Buffard et al. 2000), the real rate of measured convergence, using $L^{1}$ norm, is around 0.82 for both velocity and pressure variables when using the first order scheme (respectively, 0.98 when using a MUSCL reconstruction on primitive variables) and a bit lower (approximately $0.66)$ for density. This is due to the fact that both $U$ and $p$ remain constant through the contact discontinuity of the Euler set of equations and only vary through genuinely non-linear fields. The scheme has also been applied in order to compute the Euler equations with real gas equations-of-state, using either structured or unstructured meshes (Buffard et al. 2000). The behaviour of the scheme is also fairly good when applied to shallow-water equations, even when "vacuum" occurs in the solution or when dam-break waves are computed (Buffard et al. 1998). The extension of the scheme to non-conservative hyperbolic systems was first performed by Buffard et al. (1999).

All computations discussed below have been obtained with a CFL number equal to $1 / 2:(\Delta t / h) \max \left(\left|\lambda_{1}\right|,\left|\lambda_{8}\right|\right)=$ 0.5 . This enables to ensure that non-linear waves do not interact within each cell. In practice, almost all computations might be performed using a higher value (around 0.9 ), except probably when very strong shock waves occur or when strong double rarefaction waves develop. The unit normal vector is assumed to be tangent to the $x$-axis: $\mathbf{n}=(1,0,0)^{t}$. Thus, $U$ and $U_{n}=\mathbf{U} \cdot \mathbf{n}$ are identical here (a similar remark holds for $V$ and $U_{\tau}=\mathbf{U} \cdot \tau$ ).

We present now the numerical results obtained with the initial conditions which are similar to those pertaining to the Sod shock tube problem, considering the basic set of "laminar" variables and adding new initial conditions on each side of the initial interface for "turbulent" variables, i.e. $R_{11}, \mathrm{R}_{22}, R_{33}, R_{12}$. Figure 2 displays the computed solution of a Riemann problem associated with (10), using the basic first order time-space scheme, the following initial data

$$
\mathbf{Y}_{L}=\left(\begin{array}{c}
\rho^{-1}=1 \\
U=0 \\
V=0 \\
p=10^{5} \\
R_{11}=10^{3} \\
R_{22}=10^{3} \\
R_{12}=5 \cdot 10^{2} \\
R_{33}=10^{3}
\end{array}\right) ; \quad \mathbf{Y}_{R}=\left(\begin{array}{c}
\rho^{-1}=8 \\
U=0 \\
V=0 \\
p=10^{4} \\
R_{11}=10^{3} \\
R_{22}=10^{3} \\
R_{12}=5 \cdot 10^{2} \\
R_{33}=10^{3}
\end{array}\right),
$$

and a rather fine uniform mesh (5000 nodes in the $x$ direction). This is a difficult test case, since both the turbulent Mach numbers and the anisotropy of the Reynolds stress tensor are by no way negligible. Thus, there exists a strong coupling not only between various Reynolds stress components, but also between mean variables and second moments. The time step is in agreement with the abovementioned CFL condition.

Similar to the pure gas dynamics case, a subsonic rarefaction wave propagates to the left, and a shock wave travels to the right. The behaviour of the normal (axial) component of the velocity $U$ is quite similar to its counterpart in gas dynamics; however, the tangential (cross) velocity $V$ no longer remains null (Fig. 2b) due to the strong shear induced by the non-zero component $R_{12}$. Riemann invariants $U, p+R_{11}$ are well preserved through the 2wave, the 3-4-5-6 wave and the 7-wave (see Fig. 2a, b). It should also be emphasized that the mean density (Fig. 2c) and the Reynolds stress component $R_{11}$ (Fig. $2 \mathrm{~d}$ ) do not vary across the 2 -wave and the 7 -wave, according to the respective theoretical results detailed in Appendix A. Numerical results obtained using a coarse mesh are of very poor quality, since the two new waves (the 2 -wave and the 7-wave) can hardly be distinguished.

Some other notations are introduced in Fig. $2 \mathrm{~g}$ :

$$
V^{+}=V+\frac{R_{12}}{\left(\rho R_{11}\right)^{1 / 2}} ; \quad V^{-}=V-\frac{R_{12}}{\left(\rho R_{11}\right)^{1 / 2}} .
$$

Let us recall that $V^{+}$(respectively, $V^{-}$) is a Riemann invariant of the 2-wave (respectively, the 7-wave), see Appendices A2, A3. Figure $2 \mathrm{f}$ shows that the mean pressure $p$ varies considerably through the contact discontinuity associated with the 3-4-5-6 wave, which is a direct consequence of the presence of turbulence (this has already been pointed out by Hérard (1995a,b) and Louis (1995) when considering two-equation models). It appears clearly that the 2 -wave and the 3-4-5-6 wave can hardly be distinguished even on this rather fine mesh; this results in a rather strange behaviour of $V^{+}$due to the fact that the turbulent component $R_{11}$ on the left hand side of the contact discontinuity associated with the eigenvalues $\lambda_{3}-\lambda_{6}$ in Proposition 2 is indeed very small (as compared with the left initial value of $R_{11}$ ). All Reynolds stress components (except $R_{33}$ ) are plotted together in Fig. 2h. Figure $2 \mathrm{e}$ enables to check that the determinant $R_{11} R_{22}-\left(R_{12}\right)^{2}$ remains positive in the $(x, t)$ plane.

The next computational results presented in this section, which were also obtained on a uniform grid with 5000 nodes, are displayed in Fig. 3. The CFL number is still equal to 0.5 . The initial data, which is now

$$
\mathbf{Y}_{L}=\left(\begin{array}{c}
\rho^{-1}=1 \\
U=100 \\
V=0 \\
p=10^{5} \\
R_{11}=10^{5} \\
R_{22}=10^{4} \\
R_{12}=5 \cdot 10^{3} \\
R_{33}=10^{4}
\end{array}\right) ; \quad \mathbf{Y}_{R}=\left(\begin{array}{c}
\rho^{-1}=1 \\
U=-100 \\
V=0 \\
p=10^{5} \\
R_{11}=10^{5} \\
R_{22}=10^{4} \\
R_{12}=5 \cdot 10^{3} \\
R_{33}=10^{4}
\end{array}\right) \text {, }
$$



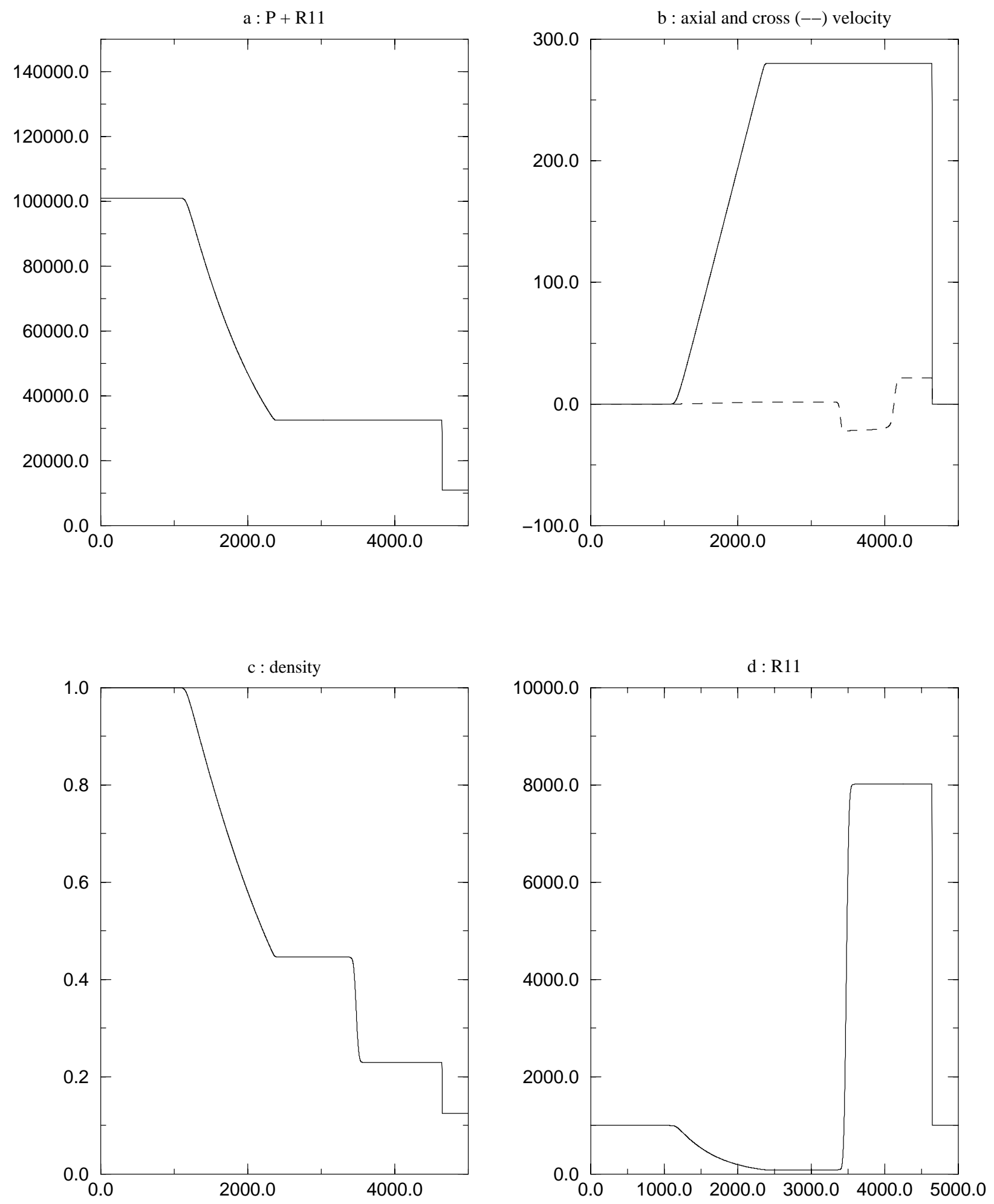

Fig. $\mathbf{2 a}-\mathbf{h}$. The first test case: a Sod-type shock tube problem 

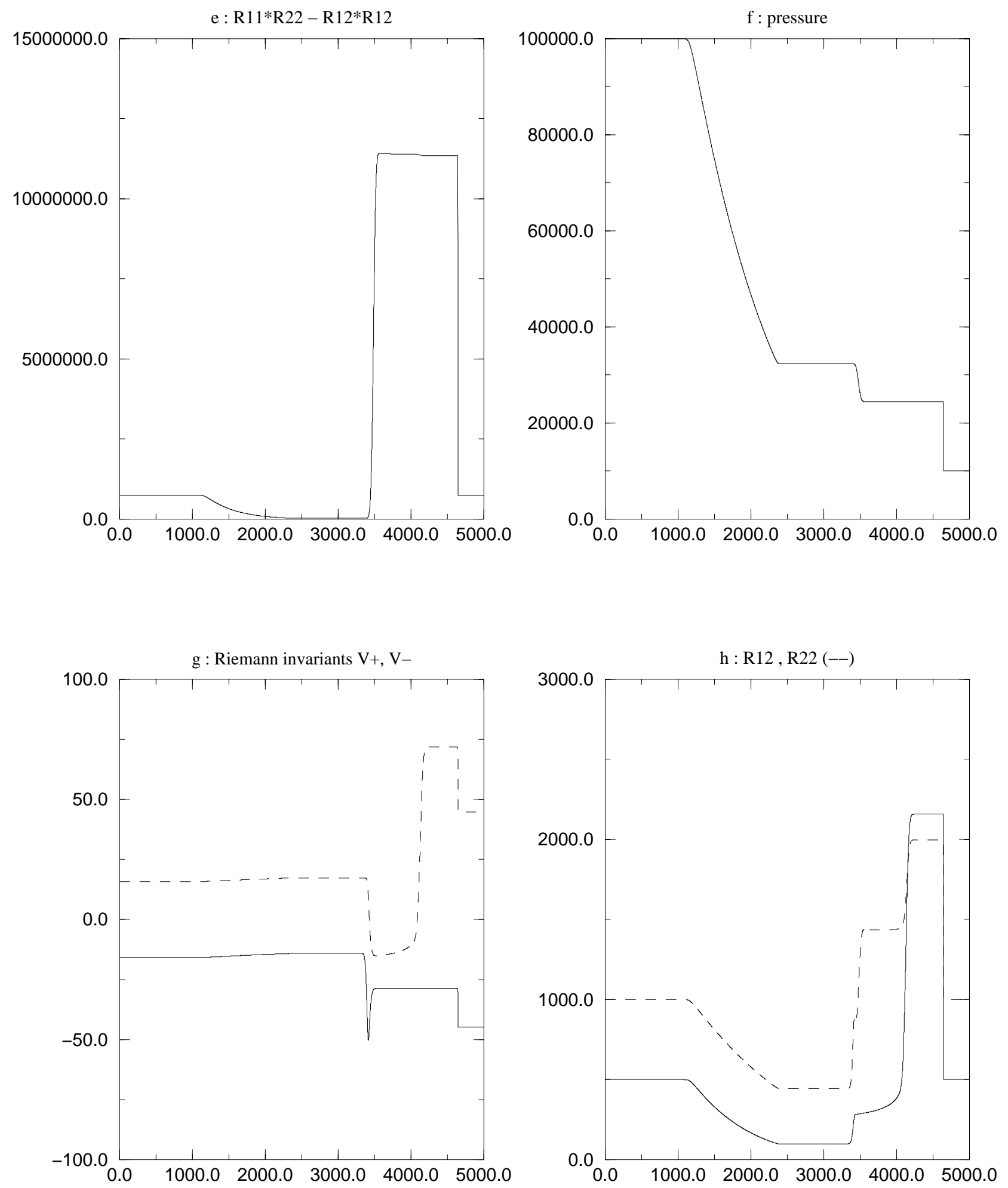

Fig. 2. (continued) 

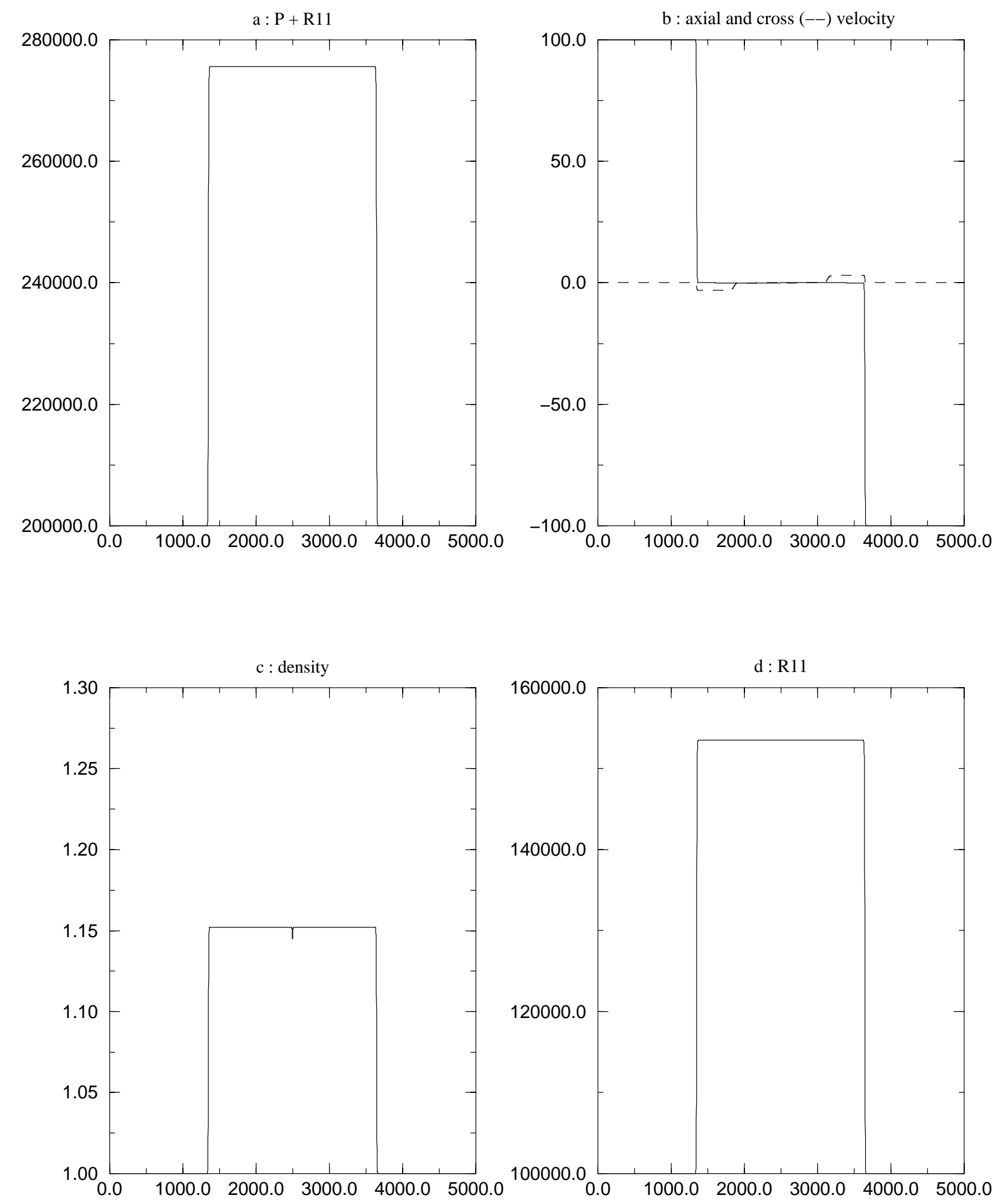

Fig. $\mathbf{3 a}-\mathbf{h}$. The second test case: a strong double shock wave 

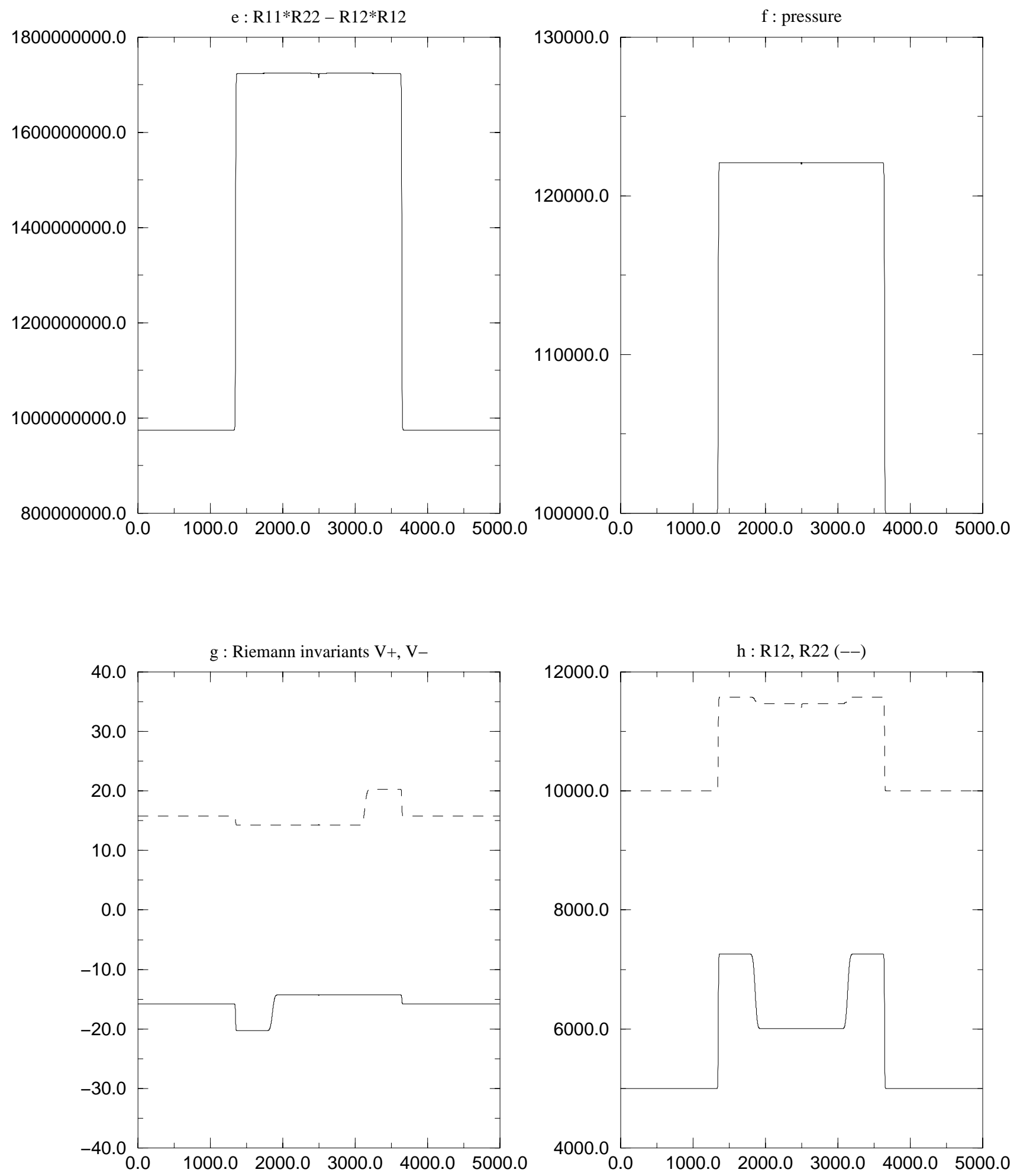

Fig. 3. (continued) 
generates a strong double shock wave. The high initial ratio $R_{11} / p$ has been chosen intentionally, in order to check the capability of the scheme to handle high turbulent Mach numbers, which seems to be compulsory when one aims at investigating the behaviour of jets impinging on wall boundaries. When looking at the predicted values of the mean density and some Reynolds stress components, a small glitch located at the position of the initial discontinuity is visible. It is due to the fact that a perturbation initially created there is hardly smoothed out since the (null) eigenvalue associated with $\lambda_{3-6}$ vanishes there during the whole computational time. This test case is indeed interesting since it enables to predict the behaviour of the scheme when computing jets impinging on wall boundaries in two- or three-dimensional geometries. This is also one of the best test cases to illustrate the failure of (uncoupled) approximate Riemann solvers based on the wave structure of the Euler set of equations (Uhlmann 1997). Results obtained with an approximate version of the Roe scheme are also briefly described in Appendix D (Fig. 6).

The third series of figures (Fig. 4) corresponds to the computation of a strong double rarefaction wave (still using a uniform grid with 5000 nodes) with the help of the VFRoe scheme of Sect.5.2. The CFL number is again equal to 0.5 , and the initial data

$$
\mathbf{Y}_{L}=\left(\begin{array}{c}
\rho^{-1}=1 \\
U=-1000 \\
V=0 \\
p=10^{5} \\
R_{11}=10^{5} \\
R_{22}=10^{3} \\
R_{12}=10^{3} \\
R_{33}=10^{3}
\end{array}\right) ; \quad \mathbf{Y}_{R}=\left(\begin{array}{c}
\rho^{-1}=1 \\
U=1000 \\
V=0 \\
p=10^{5} \\
R_{11}=10^{5} \\
R_{22}=10^{3} \\
R_{12}=10^{3} \\
R_{33}=10^{3}
\end{array}\right)
$$

generates a strong double rarefaction wave. This may represent a schematic view of the flowfield behind a bluff body. Although it is not the case here, we recall that these initial conditions may lead to the occurrence of vacuum when the modulus of the normal velocity is high enough (see the condition arising in Proposition 3 in Sect. 4). The mean axial velocity (Fig. 4b) is not a linear function of $x / t$, unless $\gamma$ is equal to 3 , since the effective celerity $c$ varies as

$$
\begin{aligned}
c & =\left(\gamma \frac{P_{L}}{\rho_{L}}\left(\frac{\rho}{\rho_{L}}\right)^{\gamma-1}+3 \frac{\left(R_{11}\right)_{L}}{\rho_{L}}\left(\frac{\rho}{\rho_{L}}\right)^{2}\right)^{1 / 2} \\
& =\left(\gamma \frac{P_{L}}{\rho_{L}}\right)^{1 / 2}\left(\frac{\rho}{\rho_{L}}\right)^{(\gamma-1) / 2}\left(1+\frac{3}{\gamma} \frac{\left(R_{11}\right)_{L}}{P_{L}}\left(\frac{\rho}{\rho_{L}}\right)^{3-\gamma}\right)^{1 / 2}
\end{aligned}
$$

in the 1-rarefaction wave (respectively, in the 8-rarefaction wave). The cross velocity is non-zero, as may be checked in Fig. 4b. The small variation of the mean density (Fig. 4c) around the initial interface is characteristic of the Godunov approach. Again, although the minimum value of the product of eigenvalues is indeed small as compared with the left (or right) values, no loss of positivity is observed. Due to the very small order of magnitude of the stress component $R_{11}$ around the characteristic line $x / t=0$, all three contact discontinuities tend to merge, as may be seen in Fig. $4 \mathrm{~g}$.

In Fig. 5 we provide some comparison between the numerical results obtained when applying the above scheme (VFRoe) and an extension of the Rusanov (1961) scheme to the framework of non-conservative systems (Appendix C), following the initial proposition by Hérard (1995b). Similar results may also be found in the work of Périgaud and Archambeau (2000). The main purpose is to demonstrate that rather simple schemes may provide meaningful results, if the overall eigenstructure is accounted for. The initial conditions of the test case are the same as those of the previous one. It is clear that both computational results are very similar. The Rusanov scheme performs in this test case rather well since neither contact discontinuity nor shock wave are present in the solution. The small density spike seen in the solution is more smeared by the extended Rusanov scheme (Fig. 5d). It may be noted that this robust scheme enables to handle vacuum occurrence (see the review on the basis of the Euler equations by Seguin 2000 and Gallouët et al. 2000).

\section{Conclusion}

The main aim of the paper is to present an approximate solution of the one-dimensional Riemann problem associated with an objective realisable second-moment closure which is valid for compressible flows. Basic analysis of the non-conservative first order differential system obviously indicates that the structure of internal waves is quite different from its counterpart in non-turbulent flows (the Euler equations). This analysis is crucial since the amount of physical diffusive effects in these turbulent models is much smaller than that in two-equation models.

An algorithm has been suggested in order to compute this second-moment closure. Numerical solutions confirm that the present finite-volume technique provides rather satisfactory results (in particular, diagonal components as well as fundamental minors, such as $R_{11} R_{22}-\left(R_{12}\right)^{2}$, remain positive). However, it becomes clear that accurate computations of the two specific "slow" waves associated with $\lambda_{2}$ and $\lambda_{7}$ require using either fine meshes or at least a second-order extension. As might have been expected, approximate Riemann solvers based on the Euler set of equations should not be used since they may provide spurious solutions (Uhlmann 1997), for instance when computing flows in the vicinity of wall boundaries, and hence are not suitable to handle computations of compressible turbulent flows using the second-order approach. The extension of the Roe-type scheme introduced by Hérard (1995b) to compute non-conservative hyperbolic systems also provides rather good results when computing single-phase turbulent compressible flows with shocks using secondmoment closures. This was recently demonstrated by Uhlmann (1997). A sample result is described in Appendix D. Moreover, the simple extension of the Rusanov scheme has also been shown to provide rather nice results (Périgaud and Archambeau 2000). 

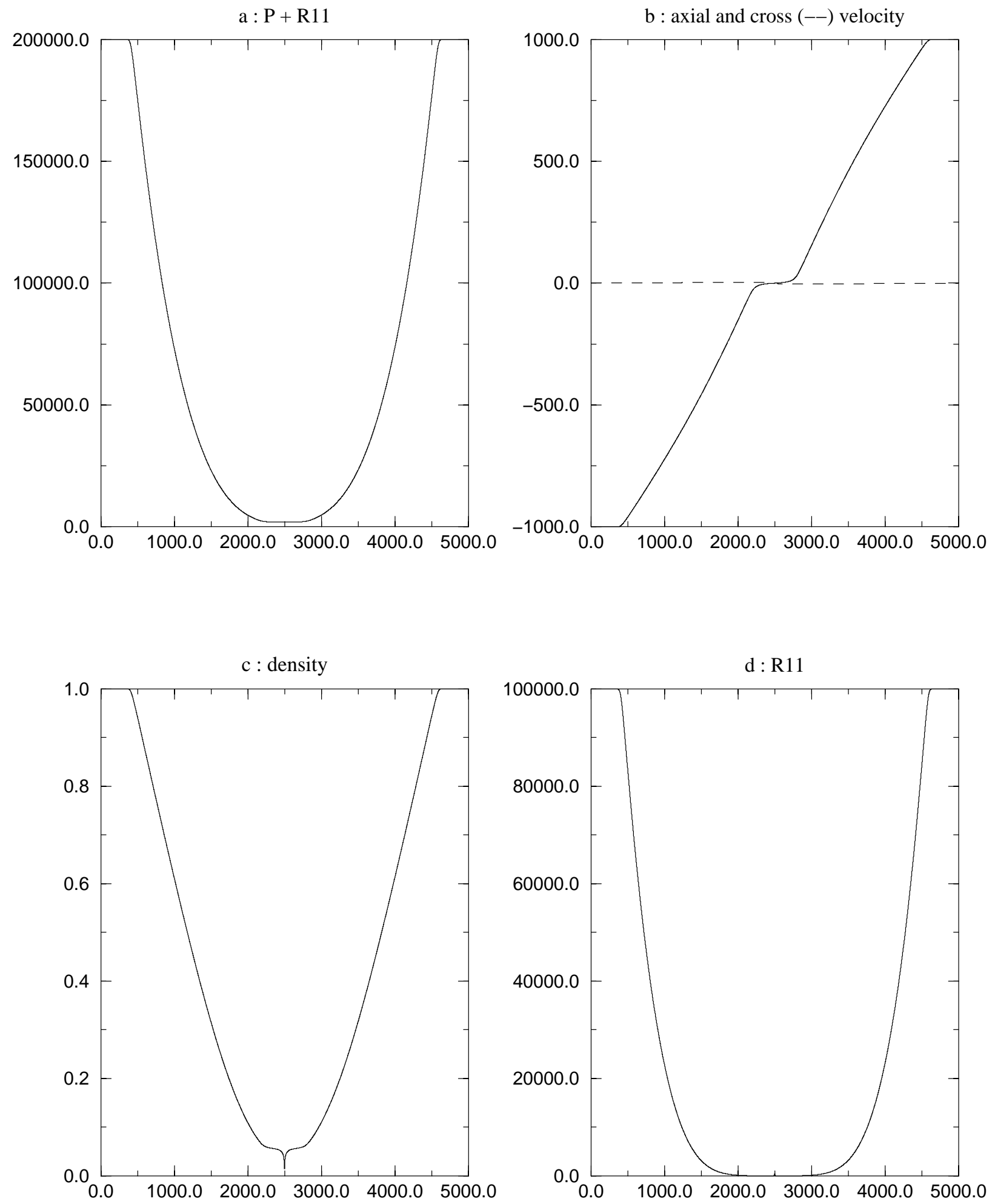

Fig. $4 \mathbf{a}-\mathbf{h}$. The third test case: a double rarefaction wave 

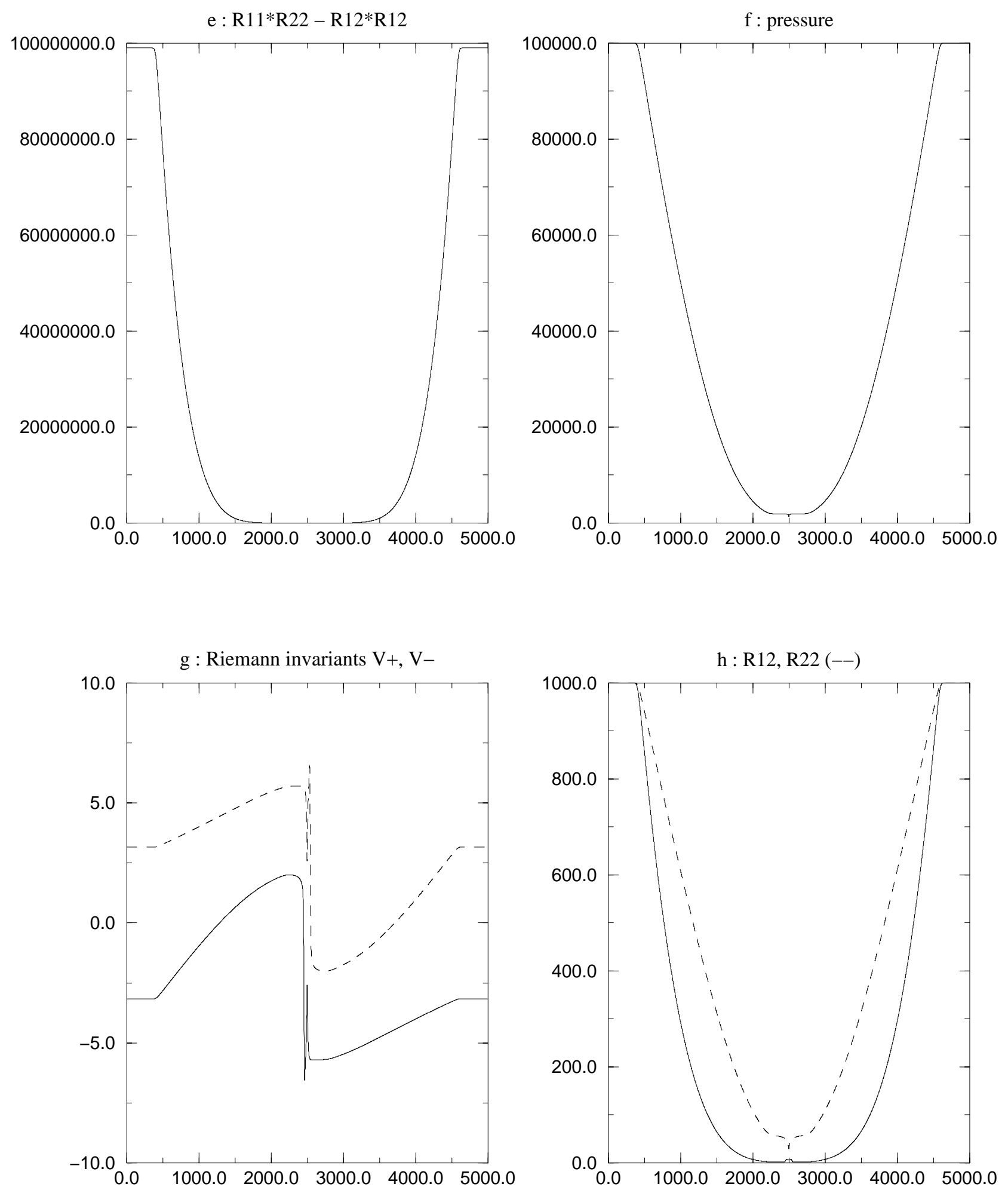

Fig. 4. (continued) 
a : axial velocity

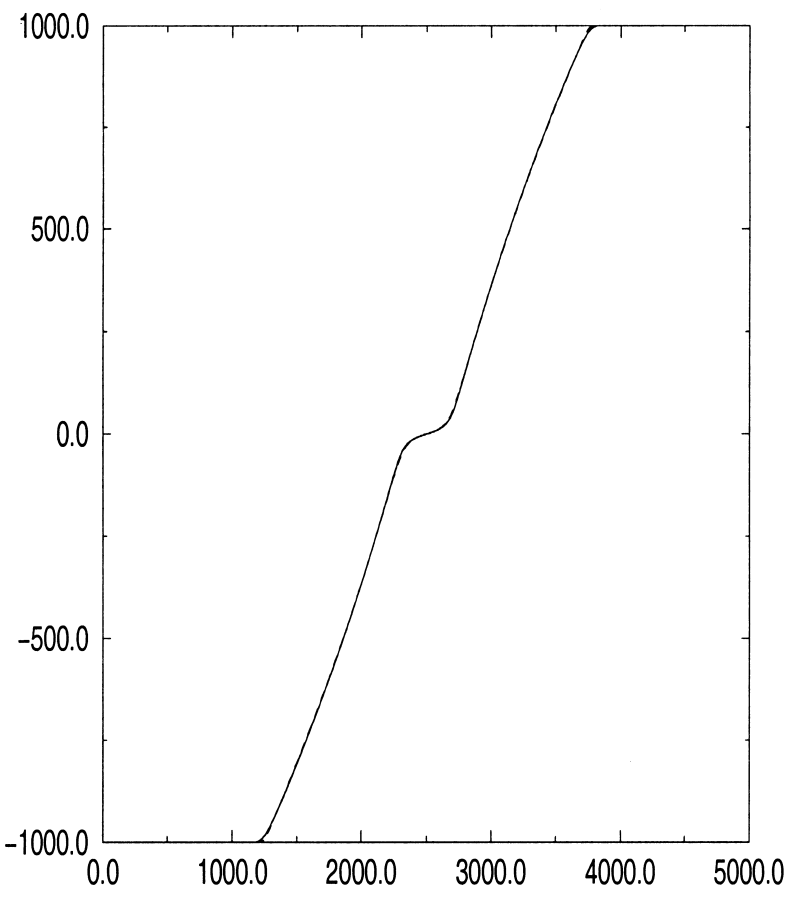

c: density

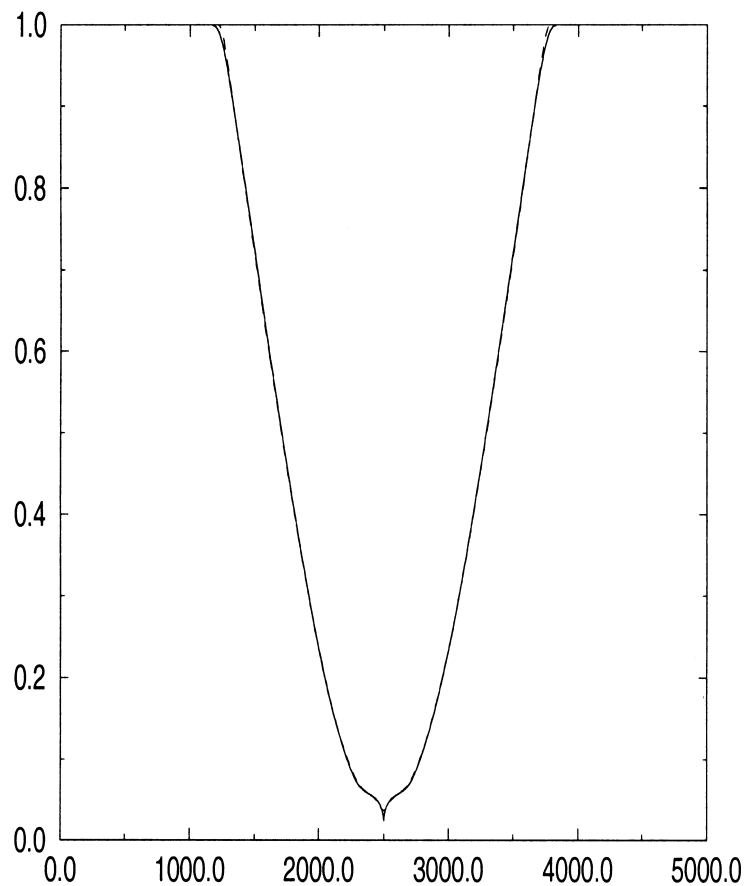

$\mathrm{b}$ : cross velocity

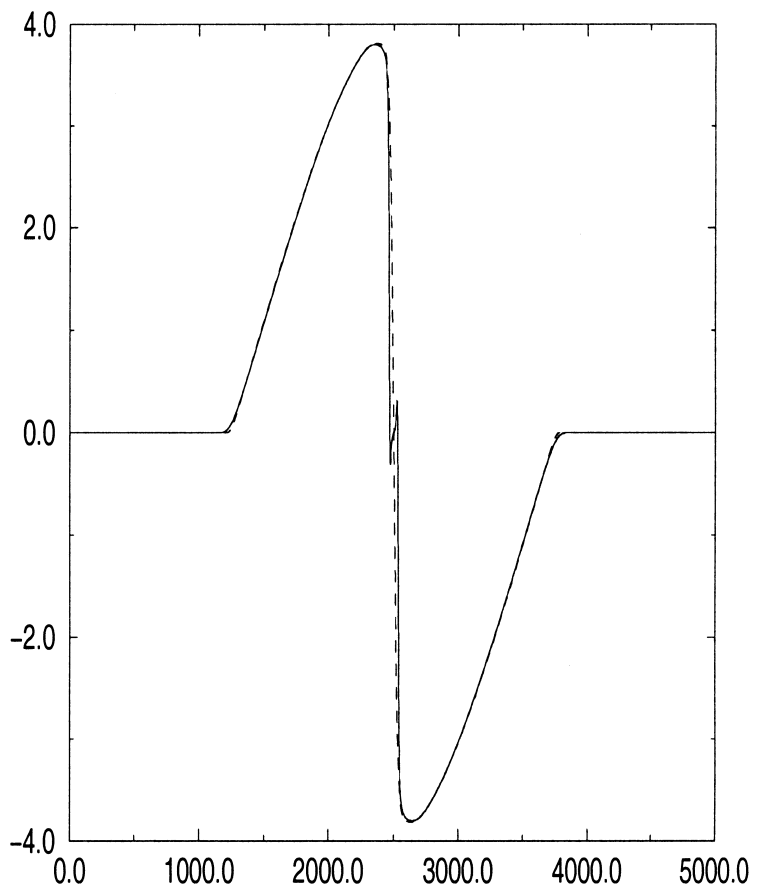

d: density

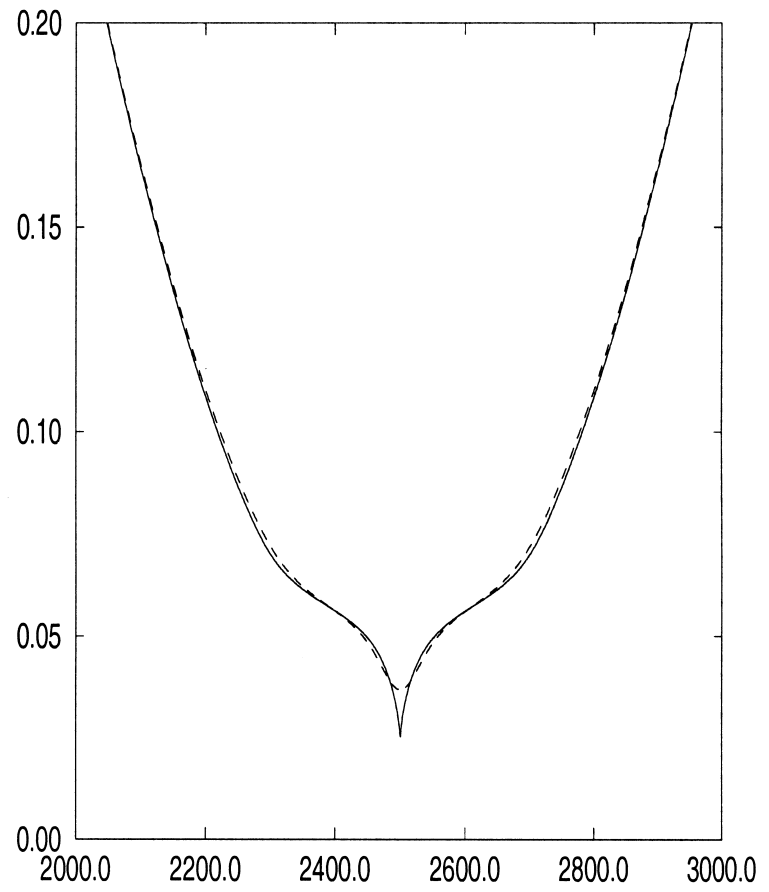

Fig. 5a-hThe third test case: a double rarefaction wave. Results for the Rusanov $(\mathbf{a}-\mathbf{f}-$ dashed lines and $\mathbf{g})$ and VFRoe $(\mathbf{a}-\mathbf{f}$ - solid lines and $\mathbf{h}$ ) schemes. Dashed and solid lines in $\mathbf{g}$ and $\mathbf{h}$ correspond to the Riemann invariants $V_{+}$and $V_{-}$, respectively 
$\mathrm{e}:$ R11

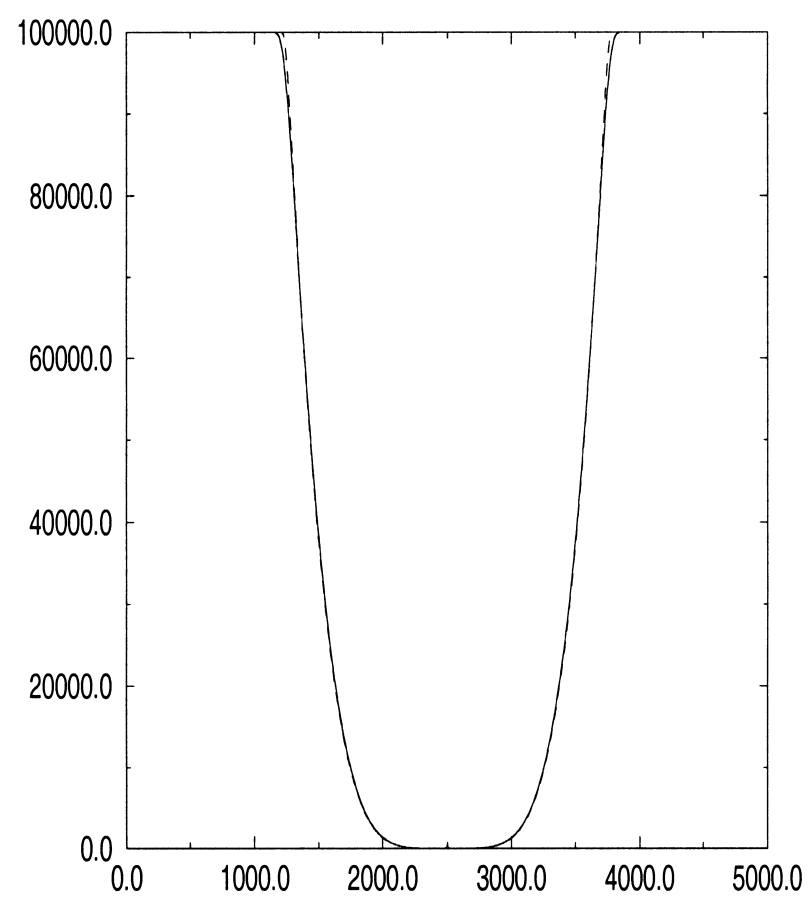

$\mathrm{g}$ : Riemann invariants (Rusanov)

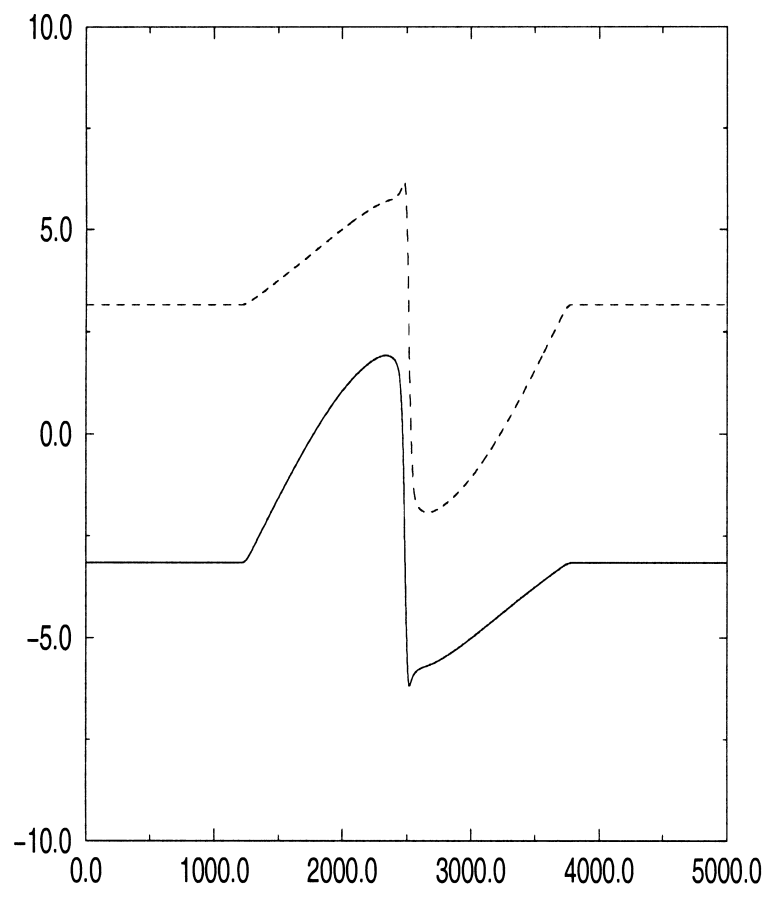

$f$ : pressure

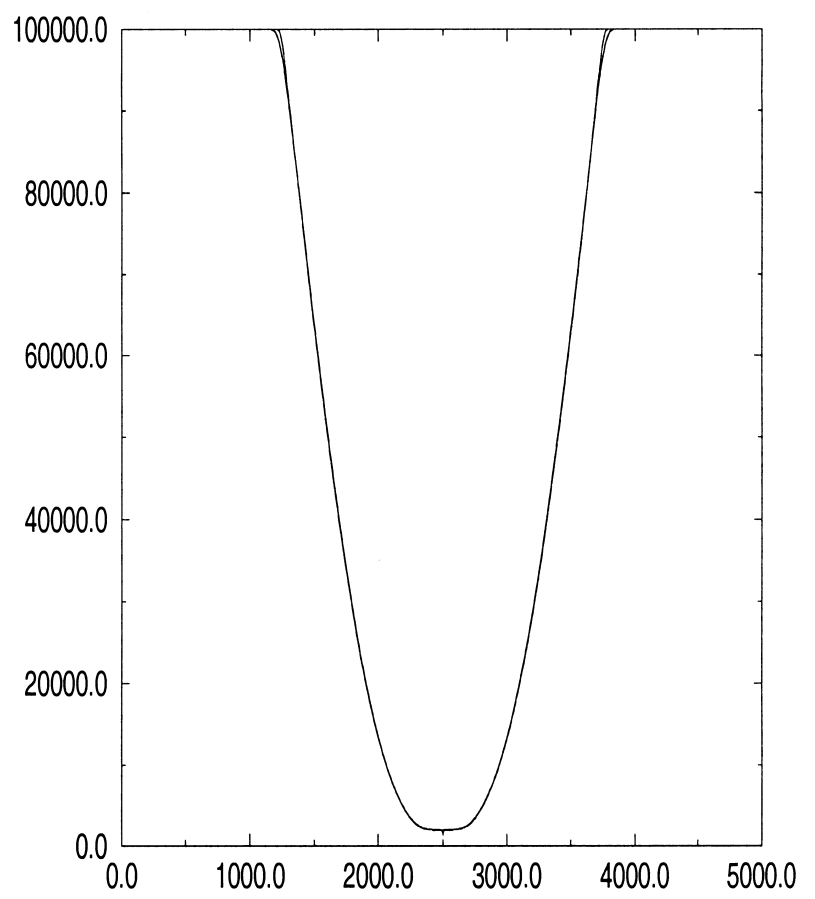

$\mathrm{h}$ : Riemann invariants (VFRoe)

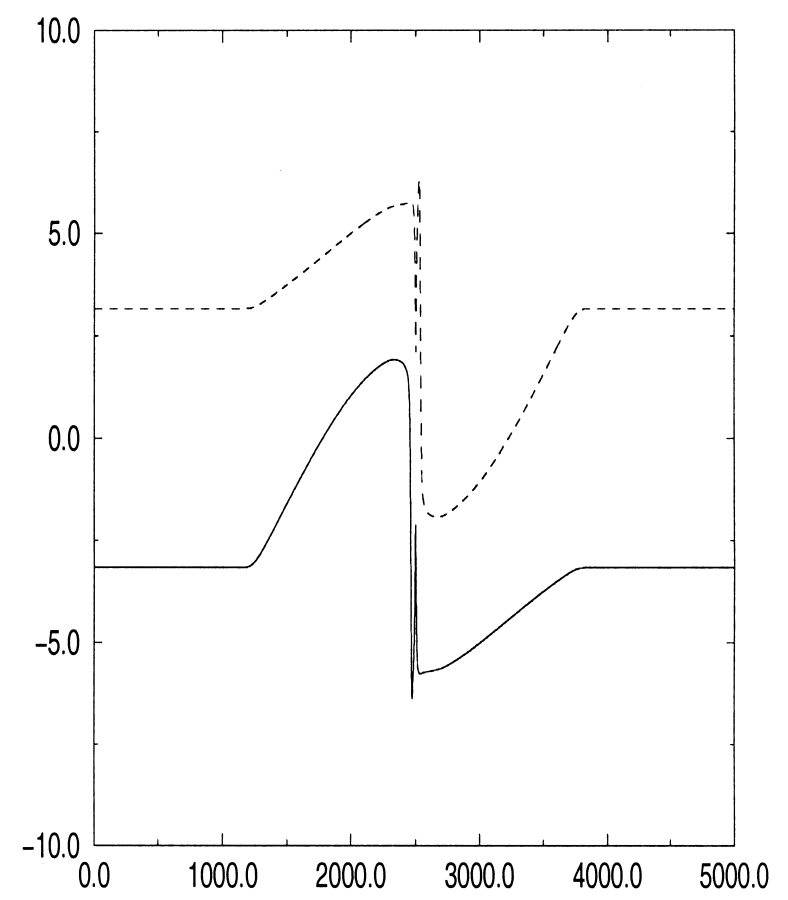

Fig. 5. (continued) 
In the present paper we were not aiming at the "ultimate" or the most well-suited scheme to deal with this non-conservative set of equations. Other extensions to the non-conservative framework of the well-known schemes such as the Osher scheme, the HLL scheme (Harten et al. 1983), or a suitable counterpart of the HLLC scheme (Toro et al. 1994; Toro 1997) in order to account for the three contact discontinuities, the AUSM scheme (Liou 1996), or other approximate Godunov schemes, such as a counterpart of PVRS scheme (Toro 1997), are certainly potentially good candidates, too.

An ongoing effort is currently directed towards the comparison of several suitable schemes for the present purpose. Obviously, a MUSCL-type second order extension of the present scheme combined with second order time integration (using the RK2 scheme, for instance) is easily feasible, using $(\rho, U, p)$ variables and Reynolds stress components. Implementation of the scheme in a finite volume code using unstructured meshes is in progress.

\section{Appendix A}

We first introduce $\tau=1 / \rho$. We also recall that

$$
p=(\gamma-1)\left(E-\frac{\rho U_{j} U_{j}}{2}-\frac{1}{2} R_{j j}\right) .
$$

The following can be then derived using the left hand sides of (1)-(4):

$$
\begin{gathered}
\tau_{, t}+U_{j} \tau_{, j}-\tau U_{j, j}=0 \\
U_{i, t}+U_{j} U_{i, j}+\tau p_{, i}+\tau R_{i j, j}=0 \\
p_{, t}+U_{j} p_{, j}+\gamma p U_{j, j}=0 ; \\
R_{i j, t}+U_{k} R_{i j, k}+R_{i j} U_{k, k}+R_{i k} U_{j, k}+R_{j k} U_{i, k}=0,
\end{gathered}
$$

when considering regular solutions. We now restrict ourselves to a two-dimensional framework, use invariance under rotation, and neglect transverse variations ( $y$-direction); hence, the local one-dimensional convective system reduces to:

$$
\begin{gathered}
\tau_{, t}+U \tau_{, x}-\tau U_{, x}=0 ; \\
U_{, t}+U U_{, x}+\tau p_{, x}+\tau R_{11, x}=0 ; \\
V_{, t}+U V_{, x}+\tau R_{12, x}=0 ; \\
p_{, t}+U p_{, x}+\gamma p U_{, x}=0 ; \\
R_{11, t}+U R_{11, x}+3 R_{11} U_{, x}=0 ; \\
R_{22, t}+U R_{22, x}+R_{22} U_{, x}+2 R_{12} V_{, x}=0 ; \\
R_{12, t}+U R_{12, x}+2 R_{12} U_{, x}+R_{11} V_{, x}=0 ; \\
R_{33, t}+U R_{33, x}+R_{33} U_{, x}=0 .
\end{gathered}
$$

\section{A1. Eigenvalues and right eigenvectors}

It may be easily checked that the eigenvalues of the matrix associated with the above-mentioned first-order differential system are

$$
\begin{gathered}
\lambda_{1}=U-c_{1} ; \quad \lambda_{2}=U-c_{2} ; \\
\lambda_{3}=\lambda_{4}=\lambda_{5}=\lambda_{6}=U ; \\
\lambda_{7}=U+c_{2} ; \quad \lambda_{8}=U+c_{1},
\end{gathered}
$$

with $c_{1}=\left(\gamma p \tau+3 \tau R_{11}\right)^{1 / 2}$ and $c_{2}=\left(\tau R_{11}\right)^{1 / 2}$.

Right eigenvectors are as follows:

$$
r_{3}=\left(\begin{array}{c}
1 \\
0 \\
0 \\
0 \\
0 \\
0 \\
0 \\
0
\end{array}\right) ; \quad r_{4}=\left(\begin{array}{c}
0 \\
0 \\
0 \\
0 \\
0 \\
0 \\
0 \\
1
\end{array}\right) ;
$$

$$
r_{5}=\left(\begin{array}{c}
0 \\
0 \\
0 \\
1 \\
-1 \\
0 \\
0 \\
0
\end{array}\right) ; \quad r_{6}=\left(\begin{array}{l}
0 \\
0 \\
0 \\
0 \\
0 \\
1 \\
0 \\
0
\end{array}\right) ;
$$

$$
r_{2}=\left(\begin{array}{c}
0 \\
0 \\
c_{2} \\
0 \\
0 \\
-2 R_{12} \\
-R_{11} \\
0
\end{array}\right) ; \quad r_{7}=\left(\begin{array}{c}
0 \\
0 \\
c_{2} \\
0 \\
0 \\
2 R_{12} \\
R_{11} \\
0
\end{array}\right)
$$




$$
r_{1}=\left(\begin{array}{c}
\tau \\
c_{1} \\
2 R_{12} c_{1}\left(\gamma p+2 R_{11}\right)^{-1} \\
-\gamma p \\
-3 R_{11} \\
-\left(R_{22}+4 R_{12}^{2}\left(\gamma p+2 R_{11}\right)^{-1}\right) \\
-2 R_{12} c_{1}^{2}\left(\gamma p+2 \tau R_{11}\right)^{-1} \\
-R_{33} \\
c_{1} \\
2 R_{12} c_{1}\left(\gamma p+2 R_{11}\right)^{-1} \\
\gamma p \\
3 R_{11} \\
R_{33} \\
2 R_{12} c_{1}^{2}\left(\gamma p \tau+2 \tau R_{11}\right)^{-1} \\
R_{22}\left(\gamma p+2 R_{11}\right)^{-1}
\end{array}\right)
$$

\section{A2. Riemann invariants}

Riemann invariants may now be computed; 1-wave (GNL - genuinely non-linear):

$$
\begin{aligned}
\phi= & \left\{s_{1}=p \tau^{\gamma}, s_{2}=R_{11} \tau^{3}, U-\int_{0}^{\tau} \frac{c_{1}\left(a, s_{1}, s_{2}\right)}{a} \mathrm{~d} a\right. \\
& \left.\left(R_{11} R_{22}-\left(R_{12}\right)^{2}\right) \tau^{4}, R_{12} \varphi, V+\theta, R_{33} \tau\right\} ;(\mathrm{A} 2.1
\end{aligned}
$$

2-wave (LD - linearly degenerate):

$$
\begin{array}{r}
\phi=\left\{\tau, U, p, R_{11}, R_{11} R_{22}-\left(R_{12}\right)^{2},\right. \\
\left.V+R_{12}\left(\rho R_{11}\right)^{-1 / 2}, R_{33}\right\} ;
\end{array}
$$

3-4-5-6-wave (LD):

$$
\phi=\left\{U, V, p+R_{11}, R_{12}\right\}
$$

7-wave (LD):

$$
\begin{array}{r}
\phi=\left\{\tau, U, p, R_{11}, R_{11} R_{22}-\left(R_{12}\right)^{2},\right. \\
\left.V-R_{12}\left(\rho R_{11}\right)^{-1 / 2}, R_{33}\right\} ;
\end{array}
$$

\section{8-wave (GNL):}

$$
\begin{aligned}
\phi= & \left\{s_{1}=p \tau^{\gamma}, s_{2}=R_{11} \tau^{3}, U+\int_{0}^{\tau} \frac{c_{1}\left(a, s_{1}, s_{2}\right)}{a} \mathrm{~d} a\right. \\
& \left.\left(R_{11} R_{22}-\left(R_{12}\right)^{2}\right) \tau^{4}, R_{12} \varphi, V-\theta, R_{33} \tau\right\}, \quad(\mathrm{A} 2.5
\end{aligned}
$$

using the following notations:

$$
\begin{gathered}
\varphi=\exp \left\{2 \int_{0}^{\tau} \frac{c_{1}^{2}\left\{a, s_{1}, s_{2}\right\}}{\left(\gamma p+2 R_{11}\right)\left\{a, s_{1}, s_{2}\right\}} \frac{\mathrm{d} a}{a^{2}}\right\} ; \\
\theta=-2 \int_{0}^{\tau} \frac{c_{1}\left\{a, s_{1}, s_{2}\right\} R_{12}}{\left(\gamma p+2 R_{11}\right)\left\{a, s_{1}, s_{2}\right\}} \frac{\mathrm{d} a}{a} .
\end{gathered}
$$

\section{A3. Shocks and contact discontinuities}

We start from:

$$
\begin{gathered}
-\sigma[\rho]+[\rho U]=0 \\
-\sigma[\rho U]+\left[\rho U^{2}+R_{11}+p\right]=0 ; \\
-\sigma[\rho V]+\left[\rho U V+R_{12}\right]=0 ; \\
-\sigma[E]+\left[U\left(E+R_{11}+p\right)\right]+\left[V R_{12}\right]=0 ; \\
-\sigma\left[R_{11}\right]+\left[U R_{11}\right]+2 \overline{R_{11}}[U]=0 ; \\
-\sigma\left[R_{22}\right]+\left[U R_{22}\right]+2 \overline{R_{12}}[V]=0 ; \\
-\sigma\left[R_{12}\right]+\left[U R_{12}\right]+\overline{R_{11}}[V]+\overline{R_{12}}[U]=0 ; \\
-\sigma\left[R_{33}\right]+\left[U R_{33}\right]=0
\end{gathered}
$$

Contact discontinuities:

(i) Through the 2-wave, we have

$$
[U]_{1}^{2}=[p]_{1}^{2}=[\rho]_{1}^{2}=\left[R_{11}\right]_{1}^{2}=0,
$$

so that

$$
\begin{aligned}
& \rho(U-\sigma)[V]_{1}^{2}+\left[R_{12}\right]_{1}^{2}=0 ; \\
& (U-\sigma)\left[R_{22}\right]_{1}^{2}+2 \overline{R_{12}}[V]_{1}^{2}=0 \\
& (U-\sigma)\left[R_{12}\right]_{1}^{2}+R_{11}[V]_{1}^{2}=0 .
\end{aligned}
$$

Hence

$$
\sigma=U-\left(R_{11} / \rho\right)^{1 / 2}
$$

and

$$
\begin{aligned}
& {[V]_{1}^{2}=-\left[\left(R_{11} \rho\right)^{-1 / 2} R_{12}\right]_{1}^{2} ;} \\
& R_{11}\left[R_{22}\right]_{1}^{2}-2 \overline{R_{12}}\left[R_{12}\right]_{1}^{2}=0 \Leftrightarrow\left[R_{11} R_{22}-\left(R_{12}\right)^{2}\right]_{1}^{2}=0 .
\end{aligned}
$$

Obviously

$$
\left[R_{33}\right]_{1}^{2}=0 .
$$

(ii) A similar result holds through the 7-wave:

$$
\begin{aligned}
& {[U]_{6}^{7}=[p]_{6}^{7}=[\rho]_{6}^{7}=\left[R_{11}\right]_{6}^{7}=0 ;} \\
& {\left[R_{33}\right]_{6}^{7}=0 ;} \\
& {[V]_{6}^{7}=\left[\left(R_{11} \rho\right)^{-1 / 2} R_{12}\right]_{6}^{7} ;} \\
& {\left[R_{11} R_{22}-\left(R_{12}\right)^{2}\right]_{6}^{7}=0 ;} \\
& \sigma=U+\left(R_{11} / \rho\right)^{1 / 2} .
\end{aligned}
$$

(iii) Through the 3-4-5-6-wave, we get:

$$
[U]_{2}^{6}=[V]_{2}^{6}=\left[p+R_{11}\right]_{2}^{6}=\left[R_{12}\right]_{2}^{6}=0 .
$$


Shocks:

(iv) Through the 1 -wave, we connect states " $L$ " and "1" using a real parameter $z(z>1)$ and $\beta=(\gamma+1) /(\gamma-1)$ :

$$
\begin{aligned}
& \rho_{1}=z \rho_{L} ; \\
& p_{1}=\left(\frac{\beta z-1}{\beta-z}\right) p_{L} \\
& \left(R_{11}\right)_{1}=\left(\frac{2 z-1}{2-z}\right)\left(R_{11}\right)_{L} \\
& U_{1}=U_{L}-(z-1) \times \\
& \times\left(\frac{\beta+1}{(\beta-z) z} \frac{p_{L}}{\rho_{L}}+\frac{3}{(2-z) z} \frac{\left(R_{11}\right)_{L}}{\rho_{L}}\right)^{1 / 2} .
\end{aligned}
$$

The speed of the 1-shock is:

$$
\sigma=\frac{1}{2}\left\{U_{1}+U_{l}+\left(\rho_{1}+\rho_{L}\right)\left(\frac{U_{L}-U_{1}}{\rho_{L}-\rho_{1}}\right)\right\} .
$$

Besides, we get:

$$
\begin{aligned}
& \qquad\left(R_{33}\right)_{1}=z\left(R_{33}\right)_{L} \\
& {\left[R_{11} R_{22}-\left(R_{12}\right)^{2}\right]_{L}^{1}} \\
& =2 \frac{[\rho]_{L}^{1}}{\rho_{L}+\rho_{1}}\left\{\left(\left(R_{11}\right)_{L}+\left(R_{11}\right)_{1}\right)\left(\left(R_{22}\right)_{L}+\left(R_{22}\right)_{1}\right)\right. \\
& \left.-\left(\left(R_{12}\right)_{L}+\left(R_{12}\right)_{1}\right)^{2}\right\}
\end{aligned}
$$

The remaining two variables $V$ and $R_{12}$ are the solutions of

$$
\begin{aligned}
& \left(\begin{array}{cc}
\overline{\rho U}_{1, L}-\sigma \bar{\rho}_{1, L} & 1 \\
\left(\bar{R}_{11}\right)_{1, L} & \bar{U}_{1, L}-\sigma+[U]_{L}^{1}
\end{array}\right)\left(\begin{array}{c}
V_{1} \\
\left(R_{12}\right)_{1}
\end{array}\right) \\
& =\left(\begin{array}{cc}
\rho U_{1, L}-\sigma \bar{\rho}_{1, L} & 1 \\
\left(\bar{R}_{11}\right)_{1, L} & \bar{U}_{1, L}-\sigma-[U]_{L}^{1}
\end{array}\right)\left(\begin{array}{c}
V_{L} \\
\left(R_{12}\right)_{L}
\end{array}\right) .
\end{aligned}
$$

(v) Through the 8-wave, we connect states "7" and " $R$ " using a real parameter $z(z<1)$ :

$$
\begin{aligned}
& \rho_{R}=z \rho_{7} \\
& p_{R}=\left(\frac{\beta z-1}{\beta-z}\right) p_{7} \\
& \left(R_{11}\right)_{R}=\left(\frac{2 z-1}{2-z}\right)\left(R_{11}\right)_{7} \\
& U_{R}=U_{7}+(z-1) \\
& \times\left(\frac{\beta+1}{(\beta-z) z} \frac{p_{7}}{\rho_{7}}+\frac{3}{(2-z) z} \frac{\left(R_{11}\right)_{7}}{\rho_{7}}\right)^{1 / 2} .
\end{aligned}
$$

The speed of the 8-shock is:

$$
\sigma=\frac{1}{2}\left\{U_{7}+U_{R}+\left(\rho_{7}+\rho_{R}\right)\left(\frac{U_{R}-U_{7}}{\rho_{R}-\rho_{7}}\right)\right\} .
$$

We also get:

$$
\left(R_{33}\right)_{R}=z\left(R_{33}\right)_{7}
$$

$$
\begin{aligned}
& {\left[R_{11} R_{22}-\left(R_{12}\right)^{2}\right]_{7}^{R}} \\
& =2 \frac{[\rho]_{7}^{R}}{\rho_{7}+\rho_{R}}\left\{\left(\left(R_{11}\right)_{7}+\left(R_{11}\right)_{R}\right)\left(\left(R_{22}\right)_{7}+\left(R_{22}\right)_{R}\right)\right. \\
& \left.-\left(\left(R_{12}\right)_{7}+\left(R_{12}\right)_{R}\right)^{2}\right\} \text {; } \\
& \left(\begin{array}{cc}
\overline{\rho U}_{7, R}-\sigma \bar{\rho}_{7, R} & 1 \\
\left(\bar{R}_{11}\right)_{7, R} & \bar{U}_{7, R}-\sigma+[U]_{7}^{R}
\end{array}\right)\left(\begin{array}{c}
V_{R} \\
\left(R_{12}\right)_{R}
\end{array}\right) \\
& =\left(\begin{array}{cc}
\overline{\rho U}_{7, R}-\sigma \bar{\rho}_{7, R} & 1 \\
\left(\overline{R_{11}}\right)_{7, R} & \bar{U}_{7, R}-\sigma-[U]_{7}^{R}
\end{array}\right)\left(\begin{array}{c}
V_{7} \\
\left(R_{12}\right)_{7}
\end{array}\right) .
\end{aligned}
$$

\section{A4. Solution of the one-dimensional Riemann problem}

First, let us notice that $U, p, \rho$ as well as $R_{11}$ remain unchanged through the 2 -wave and the 7 -wave. This may be verified using (A2.2), (A2.4), (A3.1), and (A3.6). Thus, in this subsection, we focus on the above subset of variables and omit the 2 -wave and the 7 -wave; this simply means that at first we are only looking for the values of intermediate states " 1 " and "7". Moreover, a glance at (A3.11) shows that both $U$ and $p+R_{11}$ satisfy

$$
\begin{aligned}
U_{1} & =U_{7} ; \\
\left(p+R_{11}\right)_{1} & =\left(p+R_{11}\right)_{7} .
\end{aligned}
$$

We may introduce $\left(z_{1}, z_{2}\right)$ such that

$$
\begin{aligned}
& \rho_{1}=z_{1} \rho_{L} ; \\
& \rho_{R}=z_{2} \rho_{7} .
\end{aligned}
$$

Then, owing to (A2.1), (A2.5) as well as (A3.13), (A3.14), (A3.21), (A3.22), we may parametrize both $p$ and $R_{11}$ as follows:

$$
\begin{gathered}
p_{1}=p_{L} h_{1}\left(z_{1}\right), \\
p_{R}=p_{7} h_{2}\left(z_{2}\right) ; \\
\left(R_{11}\right)_{1}=\left(R_{11}\right)_{L} \cdot g_{1}\left(z_{1}\right), \\
\left(R_{11}\right)_{R}=\left(R_{11}\right)_{7} \cdot g_{2}\left(z_{2}\right) ;
\end{gathered}
$$

using formula (A3.13), (A3.14) if $z_{1}$ is greater than 1 (respectively, (A2.1) if $z_{1}<1$ ), and formula (A3.21), (A3.22) if $z_{2}<1$ (respectively, (A2.5) if $z_{2}>1$ ). Normal velocities are linked through the following relations:

$$
\begin{aligned}
& U_{1}=U_{L}+f_{1}\left(z_{1} ; \rho_{L} ; p_{L} ;\left(R_{11}\right)_{L}\right) ; \\
& U_{R}=U_{7}+f_{2}\left(z_{2} ; \rho_{R} ; p_{R} ;\left(R_{11}\right)_{R}\right),
\end{aligned}
$$

owing to (A2.1), (A2.5), (A3.15), and (A3.23). Then, elimination of (A4.3), (A4.4), and (A4.5) and substitution into (A4.1) provides:

$$
\begin{aligned}
U_{R}-U_{L}= & f_{1}\left(z_{1} ; \rho_{L} ; p_{L} ;\left(R_{11}\right)_{L}\right) \\
& +f_{2}\left(z_{2} ; \rho_{R} ; p_{R} ;\left(R_{11}\right)_{R}\right) ;
\end{aligned}
$$




$$
\begin{gathered}
p_{L} h_{1}\left(z_{1}\right)+\left(R_{11}\right)_{L} \cdot g_{1}\left(z_{1}\right)=p_{R} h_{2}^{-1}\left(z_{2}\right) \\
+\left(R_{11}\right)_{R} \cdot g_{2}^{-1}\left(z_{2}\right) .
\end{gathered}
$$

This coupled system with two unknowns admits a unique solution $\left(z_{1}, z_{2}\right)$ in $[0, m] \times\left[m^{-1},+\infty\right]$ (where $m$ is defined as $m=\max \{2,(\gamma+1) /(\gamma-1)\})$, provided that the following condition holds:

$$
U_{R}-U_{L}<X_{L}+X_{R}
$$

with

$$
\begin{aligned}
X_{i}= & \left(\frac{\gamma p_{i}}{\rho_{i}}\right)^{1 / 2} \\
& \times \int_{0}^{\rho_{i}} \frac{\left(\frac{a}{\rho_{i}}\right)^{(\gamma-1) / 2}\left(1+3 \frac{\left(R_{11}\right)_{i}}{\gamma P_{i}}\left(\frac{a}{\rho_{i}}\right)^{3-\gamma}\right)^{1 / 2}}{a} \mathrm{~d} a,
\end{aligned}
$$

where $i$ is either " $L$ " (left state), or " $R$ " (right state). Here we indicate only the main lines of the proof. First of all, taking (A4.7) into account one may relate $z_{1}$ to $z_{2}$ :

$$
z_{1}=z_{1}\left(z_{2}\right), \quad \text { where } \frac{\mathrm{d} z_{1}\left(z_{2}\right)}{\mathrm{d} z_{2}} \leq 0 .
$$

Moreover, it may be checked that $f_{1}$ is a decreasing function of $z_{1}$, and $f_{2}$ is an increasing function of $z_{2}$. Thus, $f_{1}\left(z_{1}\left(z_{2}\right) ; \rho_{L} ; p_{L} ;\left(R_{11}\right)_{L}\right)+f_{2}\left(z_{2} ; \rho_{R} ; p_{R} ;\left(R_{11}\right)_{R}\right)$ is an increasing function of $z_{2}$. Eventually, the notion that

$$
\begin{gathered}
X_{L}=\lim _{z_{2} \rightarrow+\infty} f_{1}\left(z_{1}\left(z_{2}\right) ; \rho_{L} ; p_{L} ;\left(R_{11}\right)_{L}\right), \\
X_{R}=\lim _{z_{2} \rightarrow+\infty} f_{2}\left(z_{2} ; \rho_{R} ; p_{R} ;\left(R_{11}\right)_{R}\right)
\end{gathered}
$$

enables to conclude the proof. The reader is referred to Forestier et al. (1995), Herard (1995b), Louis (1995) for a similar result.

\section{A5. The Reynolds stress tensor is realisable}

We have to check now that Reynolds stress components satisfy the following inequalities:

$$
\begin{gathered}
R_{11} \geq 0 ; \\
R_{11} R_{22}-\left(R_{12}\right)^{2} \geq 0 ; \\
R_{33} \geq 0 .
\end{gathered}
$$

We assume that (initial) left and right states fulfill the above mentioned conditions.

A5.1. Thus, in the 1-rarefaction wave, we have, on the basis of (A2.1):

$$
\begin{gathered}
\left(R_{11}\right)_{1}=\left(R_{11}\right)_{L}\left(\frac{\tau_{L}}{\tau_{1}}\right)^{3} \geq 0 \\
\left(R_{11} R_{22}-\left(R_{12}\right)^{2}\right)_{1}=\left(R_{11} R_{22}-\left(R_{12}\right)^{2}\right)_{L}\left(\frac{\tau_{L}}{\tau_{1}}\right)^{4} \geq 0
\end{gathered}
$$

$$
\left(R_{33}\right)_{1}=\left(R_{33}\right)_{L}\left(\frac{\tau_{L}}{\tau_{1}}\right) \geq 0 .
$$

Otherwise, if the 1-wave is a shock wave, we notice that, owing to (A3.14), (A3.17) and (A3.18):

$$
\begin{gathered}
\left(R_{11}\right)_{1}=\left(\frac{2 z_{1}-1}{2-z_{1}}\right)\left(R_{11}\right)_{L} \geq 0 \\
\left(R_{33}\right)_{1}=z_{1}\left(R_{33}\right)_{L}
\end{gathered}
$$

$$
\begin{aligned}
{\left[R_{11} R_{22}-\left(R_{12}\right)^{2}\right]_{L}^{1} } & \geq 0 \Rightarrow\left(R_{11} R_{22}-\left(R_{12}\right)^{2}\right)_{1} \\
& \geq\left(R_{11} R_{22}-\left(R_{12}\right)^{2}\right)_{L} \geq 0
\end{aligned}
$$

A5.2. In the linearly degenerate 2-wave, we have, using previous results:

$$
\begin{gathered}
\left(R_{11}\right)_{2}=\left(R_{11}\right)_{1} \geq 0 ; \\
\left(R_{11} R_{22}-\left(R_{12}\right)^{2}\right)_{2}=\left(R_{11} R_{22}-\left(R_{12}\right)^{2}\right)_{1} \geq 0 ; \\
\left(R_{33}\right)_{2}=\left(R_{33}\right)_{1} \geq 0 .
\end{gathered}
$$

A5.3. Through the 8 rarefaction wave, we have, on the basis of (A2.5):

$$
\begin{aligned}
\left(R_{11}\right)_{7} & =\left(R_{11}\right)_{R}\left(\frac{\tau_{R}}{\tau_{7}}\right)^{3} \geq 0 \\
\left(R_{11} R_{22}-\left(R_{12}\right)^{2}\right)_{7} & =\left(R_{11} R_{22}-\left(R_{12}\right)^{2}\right)_{R}\left(\frac{\tau_{R}}{\tau_{7}}\right)^{4} \geq 0 ; \\
\left(R_{33}\right)_{7} & =\left(R_{33}\right)_{R}\left(\frac{\tau_{R}}{\tau_{L}}\right) \geq 0 .
\end{aligned}
$$

In the 8-shock wave, we get, using (A3.22), (A3.25), (A3.26):

$$
\begin{aligned}
\left(R_{11}\right)_{7}=\left(R_{11}\right)_{R}\left(\frac{2 z_{2}-1}{2-z_{2}}\right)^{-1} \geq 0 ; \\
\left(R_{33}\right)_{7}=\left(R_{33}\right)_{R} z_{1}^{-1} \geq 0 ; \\
{\left[R_{11} R_{22}-\left(R_{12}\right)^{2}\right]_{7}^{R} \leq 0 \Rightarrow 0 \leq\left(R_{11} R_{22}-\left(R_{12}\right)^{2}\right)_{R} } \\
\leq\left(R_{11} R_{22}-\left(R_{12}\right)^{2}\right)_{7} .
\end{aligned}
$$

A5.4. In the linearly degenerate 7-wave, we have, using results in A5.3:

$$
\begin{aligned}
\left(R_{11}\right)_{6} & =\left(R_{11}\right)_{7} \geq 0 ; \\
\left(R_{11} R_{22}-\left(R_{12}\right)^{2}\right)_{6} & =\left(R_{11} R_{22}-\left(R_{12}\right)^{2}\right)_{7} \geq 0 \\
\left(R_{33}\right)_{6} & =\left(R_{33}\right)_{7} \geq 0 .
\end{aligned}
$$

This completes the proof. 


\section{Appendix B}

B1. The four intermediate states are given as follows:

$$
\begin{gathered}
\mathbf{Y}_{1}=\mathbf{Y}_{L}+\alpha_{1} \hat{\mathbf{r}}_{1} ; \\
\mathbf{Y}_{2}=\mathbf{Y}_{L}+\alpha_{1} \hat{\mathbf{r}}_{1}+\alpha_{2} \hat{\mathbf{r}}_{2} ; \\
\mathbf{Y}_{6}=\mathbf{Y}_{R}-\alpha_{8} \hat{\mathbf{r}}_{8}-\alpha_{7} \hat{\mathbf{r}}_{7} ; \\
\mathbf{Y}_{7}=\mathbf{Y}_{R}-\alpha_{8} \hat{\mathbf{r}}_{8} .
\end{gathered}
$$

Coefficients associated with the "GNL" fields are:

$$
\begin{aligned}
& \alpha_{1}=\frac{1}{2 \hat{c}_{1}}[U]_{L}^{R}-\frac{\bar{\tau}}{2 \hat{c}_{1}^{2}}\left[p+R_{11}\right]_{L}^{R} ; \\
& \alpha_{8}=\frac{1}{2 \hat{c}_{1}}[U]_{L}^{R}+\frac{\bar{\tau}}{2 \hat{c}_{1}^{2}}\left[p+R_{11}\right]_{L}^{R}
\end{aligned}
$$

with

$$
\hat{c}_{1}^{2}=\bar{\tau}\left(\gamma \bar{p}+3 \overline{R_{11}}\right)
$$

and

$$
\hat{r}_{1}=\left(\begin{array}{c}
\bar{\tau} \\
\hat{c}_{1} \\
2 \bar{R}_{12} \hat{c}_{1}\left(\gamma \bar{p}+2 \bar{R}_{11}\right)^{-1} \\
-\gamma \bar{p} \\
-3 \bar{R}_{11} \\
-\left(\bar{R}_{22}+4 \bar{R}_{12}^{2}\left(\gamma \bar{p}+2 \bar{R}_{11}\right)^{-1}\right) \\
-2 \bar{R}_{12} \hat{c}_{1}^{2}\left(\gamma \bar{p} \bar{\tau}+2 \bar{\tau} \bar{R}_{11}\right)^{-1} \\
-\bar{R}_{33} \\
-\bar{\tau} \\
\hat{c}_{1} \\
\left(\gamma \bar{p}+2 \bar{R}_{11}\right)^{-1} \\
\gamma \bar{p} \\
3 \bar{R}_{11} \\
2 \bar{R}_{12} \hat{c}_{1}\left(\bar{R}_{12}^{2}\left(\gamma \bar{p}+2 \bar{R}_{11}\right)^{-1}\right. \\
\bar{R}_{22}+4 \bar{R}_{12}^{2}\left(\gamma \bar{p} \bar{\tau}+2 \bar{\tau} \bar{R}_{11}\right)^{-1} \\
\bar{R}_{33} \\
2 \bar{R}_{12} \hat{c}_{1}^{2}
\end{array}\right) ;
$$

Coefficients associated with the two new "LD" fields are:

$$
\begin{gathered}
\alpha_{2}=\frac{1}{2 \bar{R}_{11}}\left\{\frac{2 \bar{R}_{12}}{\gamma \bar{p}+2 \bar{R}_{11}}\left(\frac{-\hat{c}_{2}}{\bar{\tau}}[U]_{L}^{R}+\left[p+R_{11}\right]_{L}^{R}\right)\right. \\
\left.+\frac{\hat{c}_{2}}{\bar{\tau}}[V]_{L}^{R}-\left[R_{12}\right]_{L}^{R}\right\} ; \\
\alpha_{7}=\frac{1}{2 \bar{R}_{11}}\left\{\frac{-2 \bar{R}_{12}}{\gamma \bar{p}+2 \bar{R}_{11}}\left(\frac{\hat{c}_{2}}{\bar{\tau}}[U]_{L}^{R}+\left[p+R_{11}\right]_{L}^{R}\right)\right. \\
\left.+\frac{\hat{c}_{2}}{\bar{\tau}}[V]_{L}^{R}+\left[R_{12}\right]_{L}^{R}\right\},
\end{gathered}
$$

noting that

$$
\hat{c}_{2}^{2}=\bar{\tau} \overline{R_{11}}
$$

and

$$
\hat{r}_{2}=\left(\begin{array}{c}
0 \\
0 \\
\hat{c}_{2} \\
0 \\
0 \\
-2 \bar{R}_{12} \\
-\bar{R}_{11} \\
0
\end{array}\right) ; \quad \hat{r}_{7}=\left(\begin{array}{c}
0 \\
0 \\
\hat{c}_{2} \\
0 \\
0 \\
2 \bar{R}_{12} \\
\bar{R}_{11} \\
0
\end{array}\right) .
$$

B2. Considering, first, the 2-wave, and owing to the fact that

$$
\mathbf{Y}_{2}=\mathbf{Y}_{1}+\alpha_{2} \hat{\mathbf{r}}_{2},
$$

we immediately obtain that the numerical (approximate) intermediate states satisfy

$$
[U]_{1}^{2}=[p]_{1}^{2}=[\rho]_{1}^{2}=\left[R_{11}\right]_{1}^{2}=0,
$$

as they do in the continuous case. A similar result holds for the 7 -wave:

$$
[U]_{6}^{7}=[p]_{6}^{7}=[\rho]_{6}^{7}=\left[R_{11}\right]_{6}^{7}=0 .
$$

Turning then to the 3-4-5-6 wave, we have that the numerical values of the intermediate states "2" and "6" agree with

$$
[U]_{2}^{6}=\left[p+R_{11}\right]_{2}^{6}=[V]_{2}^{6}=\left[R_{12}\right]_{2}^{6}=0 .
$$

Hence, all these Riemann invariants are numerically preserved.

\section{Appendix C}

We briefly present here the extension of the Rusanov scheme which has been used to compute the non-conservative system

$$
\mathbf{Z}_{, t}+\left(\mathbf{F}_{1}(\mathbf{Z})\right)_{, x}+\mathbf{A}_{1}^{\mathrm{nc}}(\mathbf{Z}) \mathbf{Z}_{, x}=0 .
$$

The integration over the cell $\Omega_{i}$ and the constant time step $\Delta t$ provides:

$$
\begin{aligned}
& \operatorname{vol}\left(\Omega_{i}\right)\left(\mathbf{Z}_{i}^{n+1}-\mathbf{Z}_{i}^{n}\right)+\Delta t \int_{\Gamma_{i}} \mathbf{F}_{1}^{\text {Rusanov }}\left(\mathbf{Z}^{n}\right) \mathrm{d} \Gamma \\
& \quad+\Delta t \pi_{i}\left(\mathbf{Z}^{n}\right)=0
\end{aligned}
$$

where

$$
\begin{aligned}
\mathbf{F}_{1}^{\text {Rusanov }}\left(\mathbf{Z}^{n}\right)= & \left\{\mathbf{F}_{1}\left(\mathbf{Z}_{i}^{n}\right)+\mathbf{F}_{1}\left(\mathbf{Z}_{i+1}^{n}\right)\right. \\
& \left.-r_{i+1 / 2}\left(\mathbf{Z}_{i+1}^{n}-\mathbf{Z}_{i}^{n}\right)\right\} / 2 .
\end{aligned}
$$


$a: P+R 11$

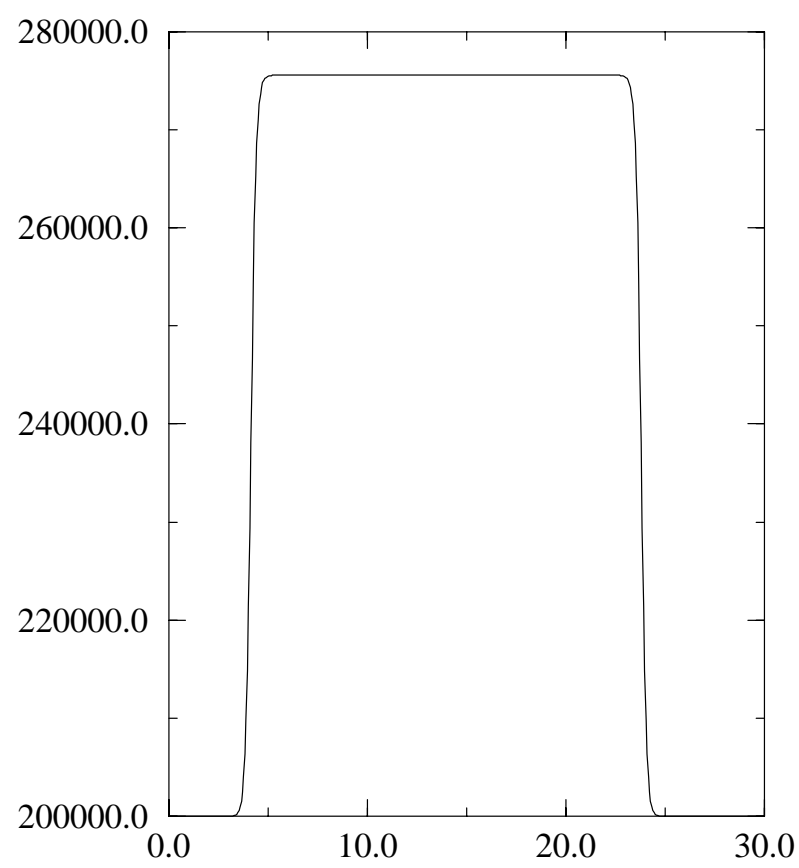

$\mathrm{b}:$ axial and cross (---) velocity

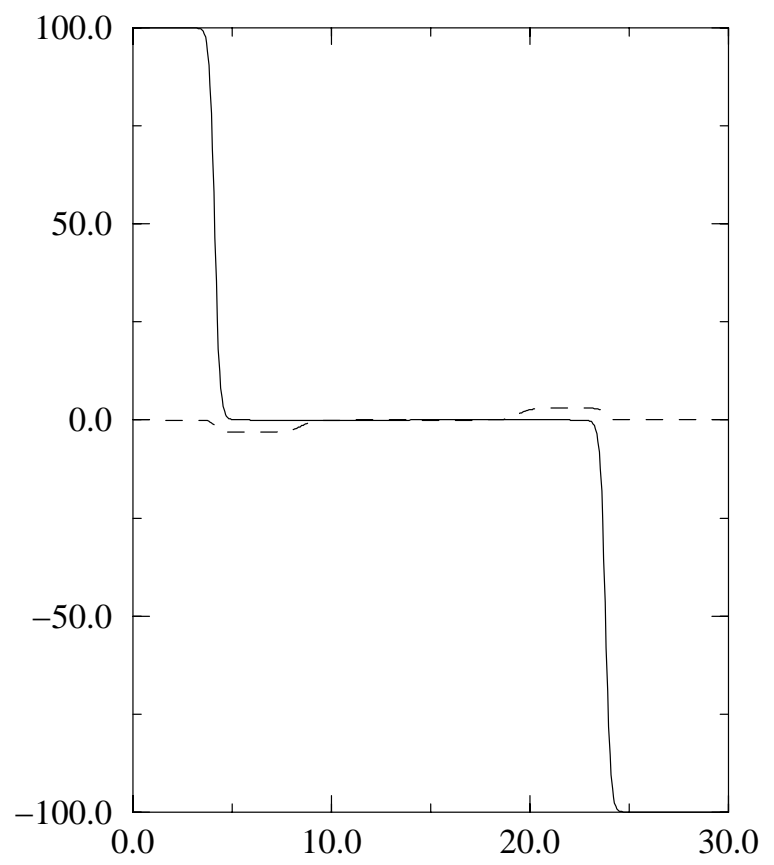

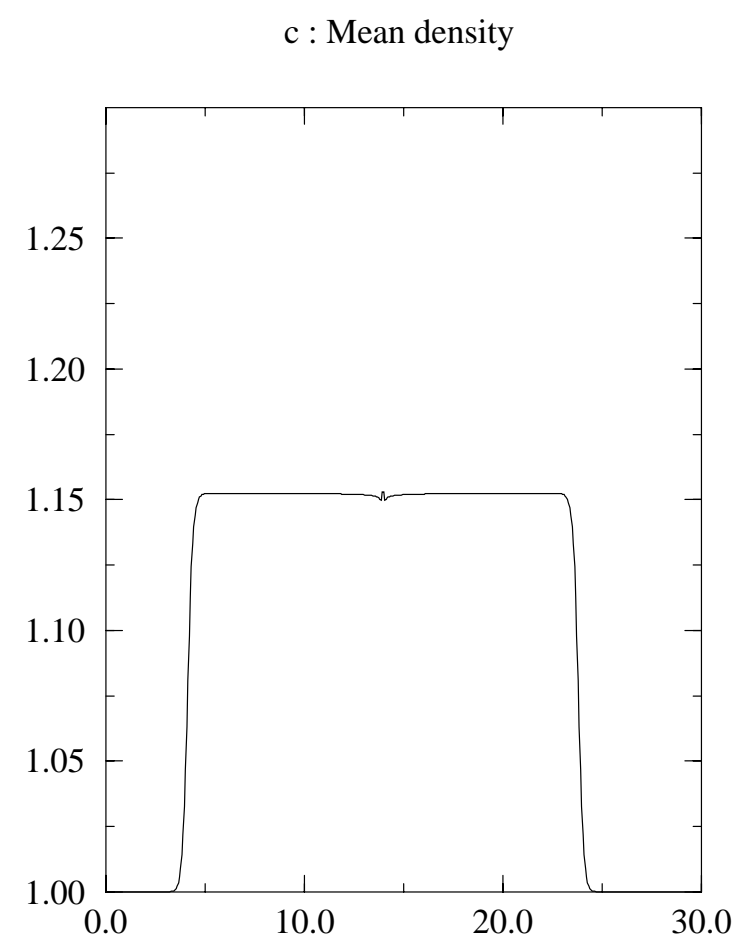

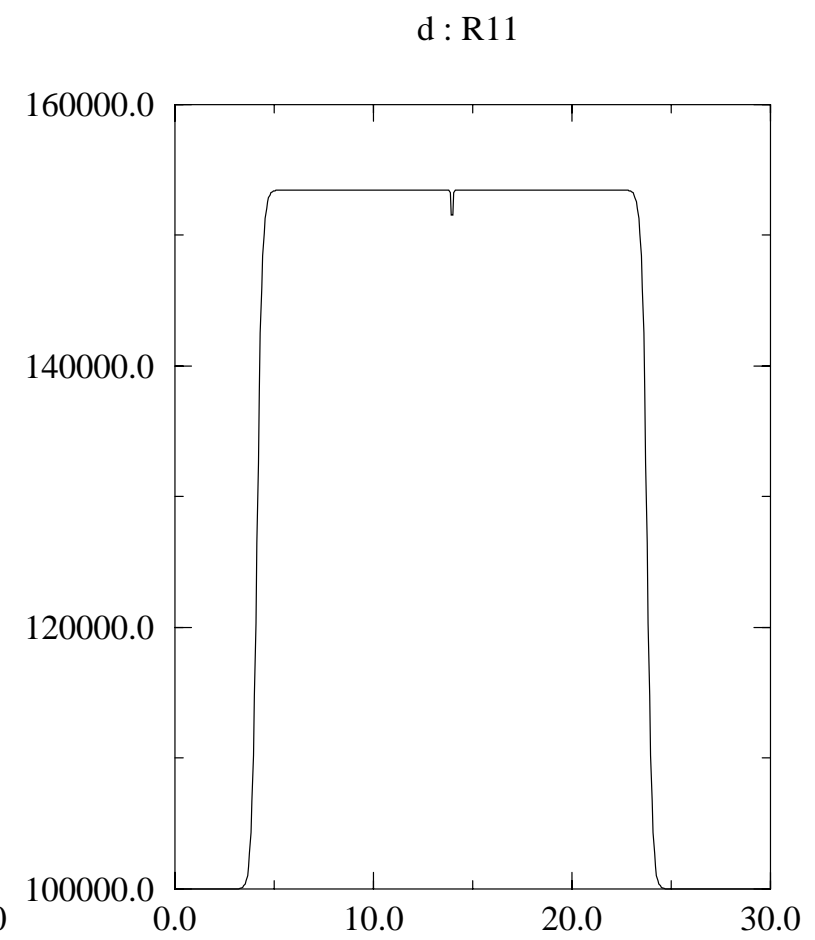

Fig. 6a-d. The second test case: a strong double shock wave. Results for the extension of the Roe scheme to the non-conservative system 
We recall that the continuous flux is given by:

$$
\mathbf{F}_{1}(\mathbf{Z})=\left(\begin{array}{c}
\rho U \\
\rho U^{2}+p+R_{11} \\
\rho U V+R_{12} \\
U\left(E+p+R_{11}\right)+V R_{12} \\
U R_{11} \\
U R_{22} \\
U R_{12} \\
U R_{33}
\end{array}\right) .
$$

The spectral radius rspec $\left(\mathbf{B}_{1}\right)$ of $\mathbf{B}_{1}(\mathbf{Z})=\mathrm{d} \mathbf{F}_{1}(\mathbf{Z}) / \mathrm{d} \mathbf{Z}+$ $\mathbf{A}_{1}^{\mathrm{nc}}(\mathbf{Z})$ is computed on each side of the interface and the resulting coefficient is written as

$$
r_{i+1 / 2}=\max \left\{\operatorname{rspec}\left(\left(\mathbf{B}_{1}\right)_{i}\right) \operatorname{rspec}\left(\left(\mathbf{B}_{1}\right)_{i+1}\right)\right\} .
$$

Integration of the non-conservative part leads to:

$$
\begin{aligned}
& \pi_{i}\left(\mathbf{Z}^{n}\right)= \\
& \left(\begin{array}{c}
0 \\
0 \\
0 \\
0 \\
2\left(R_{11}\right)_{i}\left(\bar{U}_{i+1 / 2}-\bar{U}_{i-1 / 2}\right) \\
2\left(R_{12}\right)_{i}\left(\bar{V}_{i+1 / 2}-\bar{V}_{i-1 / 2}\right) \\
\left(R_{11}\right)_{i}\left(\bar{V}_{i+1 / 2}-\bar{V}_{i-1 / 2}\right)+\left(R_{12}\right)_{i}\left(\bar{U}_{i+1 / 2}-\bar{U}_{i-1 / 2}\right) \\
0
\end{array}\right) .
\end{aligned}
$$

\section{Appendix D}

We briefly present here the extension of the Roe scheme which has been used to compute the non-conservative system

$$
\mathbf{Z}_{, t}+\left(\mathbf{F}_{1}(\mathbf{Z})\right)_{, x}+\mathbf{A}_{1}^{\mathrm{nc}}(\mathbf{Z}) \mathbf{Z}_{, x}=0 .
$$

We still denote the constant time step as $\Delta t$. The straightforward integration over cell $\Omega_{i}$ again provides

$$
\begin{aligned}
& \operatorname{vol}\left(\Omega_{i}\right)\left(\mathbf{Z}_{i}^{n+1}-\mathbf{Z}_{i}^{n}\right)+\Delta t \int_{\Gamma_{i}} \mathbf{F}_{1}^{\mathrm{Roe}}\left(\mathbf{Z}^{n}\right) \mathrm{d} \Gamma \\
& \quad+\Delta t \pi_{i}\left(\mathbf{Z}^{n}\right)=0
\end{aligned}
$$

where

$$
\begin{aligned}
\mathbf{F}_{1}^{\mathrm{Roe}}\left(\mathbf{Z}^{n}\right)= & \left(\mathbf{F}_{1}\left(\mathbf{Z}_{i}^{n}\right)+\mathbf{F}_{1}\left(\mathbf{Z}_{i+1}^{n}\right)\right. \\
& -\Omega\left(\mathbf{Z}\left(\overline{\mathbf{Y}}_{i+1 / 2}\right)\right)|\wedge|\left(\mathbf{Z}\left(\overline{\mathbf{Y}}_{i+1 / 2}\right)\right) \\
& \left.\Omega^{-1}\left(\mathbf{Z}\left(\overline{\mathbf{Y}}_{i+1 / 2}\right)\right)\left(\mathbf{Z}_{i+1}^{n}-\mathbf{Z}_{i}^{n}\right)\right) / 2 .
\end{aligned}
$$

The state $\mathbf{Y}$ is the same as in the main text: $\mathbf{Y}^{t}=\left(\rho^{-1}\right.$, $\left.U, V, p, R_{11}, R_{22}, R_{12}, R_{33}\right)$. The continuous flux is still given by

$$
\mathbf{F}_{1}(\mathbf{Z})=\left(\begin{array}{c}
\rho U \\
\rho U^{2}+p+R_{11} \\
\rho U V+R_{12} \\
U\left(E+p+R_{11}\right)+V R_{12} \\
U R_{11} \\
U R_{22} \\
U R_{12} \\
U R_{33}
\end{array}\right) .
$$

$\Lambda(\mathbf{Z})$ and $\Omega(\mathbf{Z})$ represent the diagonal matrix of eigenvalues and the matrix of right eigenvectors of the matrix of convective effects $\mathbf{B}_{1}(\mathbf{Z})=\mathrm{d} \mathbf{F}_{1}(\mathbf{Z}) / \mathrm{d} \mathbf{Z}+\mathbf{A}_{1}^{\text {nc }}(\mathbf{Z})$, respectively. The integration of the non-conservative part $\pi_{i}\left(\mathbf{Z}^{n}\right)$ is exactly the same as in Appendix $\mathrm{C}$ related to the Rusanov scheme.

The initial conditions associated with the computational results displayed in Fig. 6 are those of the Riemann problem which corresponds to a double shock wave (see the main text). Hence:

$$
\begin{aligned}
\mathbf{Y}_{L}^{t} & =\left(1,100,0,10^{5}, 10^{5}, 10^{4}, 5 \cdot 10^{3}, 10^{4}\right) ; \\
\mathbf{Y}_{R}^{t} & =\left(1,-100,0,10^{5}, 10^{5}, 10^{4}, 5 \cdot 10^{3}, 10^{4}\right) .
\end{aligned}
$$

The uniform mesh contains only 500 nodes. The CFL number is equal to 0.5 .

Acknowledgements. The authors would like to thank the reviewers for their useful remarks. The part of the work concerning the VFRoe scheme has benefited from fruitful discussions with Dr. Thierry Buffard (Université Blaise Pascal), Dr. Isabelle Faille (IFP), Prof. Thierry Gallouët (Université de Provence) and Dr. Jean-Marie Masella (IFP), who are greatly acknowledged herein. Frédéric Archambeau (EDF-DRD) and Guillaume Périgaud are also acknowledged for their help.

\section{References}

Buffard T, Gallouët T, Hérard JM (1998) A naïve scheme to compute shallow water equations. C. R. Acad. Sci., Paris, I-326, pp. 385-390

Buffard T, Gallouët T, Hérard JM (1999) A naïve Riemann solver to compute a non-conservative hyperbolic system. Int. Series on Numerical Mathematics 129: 129-138

Buffard T, Gallouët T, Hérard JM (2000) A sequel to a rough Godunov scheme: application to real gases. Computers and Fluids 29(7): 813-847

Dal Maso G, Le Floch PG, Murat F (1995) Definition and weak stability of non-conservative products. J. Math. Pures Appl. 74: 483-548

Declercq-Xeuxet E (1999) Comparaison de solveurs numériques pour le traitement de la turbulence bi fluide. $\mathrm{PhD}$ thesis, Université Evry Marne la Vallée, Paris, France, June 23 
Eymard R, Gallouët T, Herbin R (2001) Finite volume methods. In: Ciarlet PG, Lions PL (eds) Handbook for numerical analysis, Vol. 7, pp. 729-1020. North Holland

Faucher E (2000) Simulation numérique des écoulements unidimensionnels instationnaires d'écoulements avec autovaporisation. PhD thesis, Université Paris Val de Marne, Paris, France, January 24

Favre A (1965) Equations des gaz turbulents compressibles. Jour. Mecanique 4: 391-421

Forestier A, Hérard JM, Louis X (1995) Exact or approximate Riemann solvers to compute a two-equation turbulent compressible model. In: Cecchi M, Morgan K, Periaux J, Schrefler BA, Zienkiewicz OC (eds) Finite elements in fluids. New trends and applications, vol. I, pp. 677-686

Forestier A, Hérard JM, Louis X (1997) A Godunov type solver to compute turbulent compressible flows. C. R. Acad. Sci., Paris, I-324, pp. 919-926

Fu S, Launder BE, Tselepidakis DP (1987) Accomodating the effects of high strain rates in modelling the pressure strain correlations. UMIST report TFD $87 / 5$

Gallouët T, Masella JM (1996) A rough Godunov scheme. C. R. Acad. Sci., Paris, I-323, pp. 77-84

Gallouët T, Hérard JM, Seguin N (2000) Some recent finite volume schemes to compute Euler equations with real gas equations of state. Internal report LATP 00-21, Université de Provence, Marseille, France

Godlewski E, Raviart PA (1996) Numerical analysis of hyperbolic systems of conservation laws. Springer Verlag

Godunov SK (1959) A difference method for numerical calculation of discontinuous equations of hydrodynamics. Math. Sb. 47: 217-300

Harten A, Lax PD, Van Leer B (1983) On upstream differencing and Godunov type schemes for hyperbolic conservation laws. SIAM Review 25(1): 35-61

Hérard JM (1994) Basic analysis of some second moment closures. Part I: incompressible isothermal turbulent flows. Theoretical and Computational Fluid Dynamics 6(4): 213233

Hérard JM (1995a) Suitable algorithms to preserve the realisability of Reynolds stress closures. ASME Fluids Engineering Division 215: 73-80

Hérard JM (1995b) Solveur approché pour un système hyperbolique non conservatif issu de la turbulence compressible. EDF report HE-41/95/009/A, Electricité de France, Chatou, France

Hérard JM (1996) Realisable non-degenerate second moment closures for incompressible turbulent flows. C. R. Acad. Sci., Paris, t.322, IIb, pp. 371-377

Hérard JM, Forestier A, Louis X (1995) A non-strictly hyperbolic system to describe compressible turbulence. Collection des Notes internes de la Direction des Etudes et Recherches d'EDF n 95NB00003, Electricité de France, Chatou, France

Le Floch P (1988) Entropy weak solutions to non-linear hyperbolic systems in non-conservative form. Comm. in Part. Diff. Eq. 13(6): 669-727

Le Floch P, Liu TP (1992) Existence theory for non-linear hyperbolic systems in non-conservative form. CMAP report 254, Ecole Polytechnique, Palaiseau, France

Liou M (1996) A sequel to AUSM: AUSM +. J. Comp. Physics 12(2): $364-382$

Louis X (1995) Modélisation numérique de la turbulence compressible. PhD thesis, Université Paris VI, Paris, France, July 8
Lumley JL (1978) Computational modelling of turbulent flows. Advances in Applied Mechanics 18: 123-176

Lumley JL (1983) Turbulence modelling. J. of Applied Mechanics 50: 1097-1103

Masella JM (1997) Quelques méthodes numériques pour les écoulements diphasiques bi-fluide en conduites pétrolières. PhD thesis, Université Paris VI, Paris, France, May 5

Masella JM, Faille I, Gallouët T (1999) On a rough Godunov scheme. Int. J. for Computational Fluid Dynamics 12(2): $133-150$

Page A, Uhlmann M (1996) Traitement de la partie hyperbolique du système des équations de Navier-Stokes moyénnées et des équations de transport issues d'une femeture au premier ordre pour un fluide compressible. Internal report 96-1, LMFA, Ecole Centrale de Lyon, Lyon, France

Périgaud G, Archambeau F (2000) Méthodes non conservatives multidimensionnelles pour modèle de turbulence compressible au second ordre. Internal EDF report HI-83/00/028/A, Electricité de France, Chatou, France

Pope SB (1985) PDF methods for turbulent reactive flows. Prog. Energy Combust. Sci. 11: 119-192

Ristorcelli J (1993) A representation for the turbulent mass flux contribution to Reynolds stress and two-equation closures for compressible turbulence. NASA ICASE Technical Report 93-88

Roe PL (1981) Approximate Riemann solvers, parameter vectors and difference schemes. J. Comp. Physics 43: 357-372

Rusanov VV (1961) Calculation of interaction of non steady shock waves with obstacles. J. Comp. Math. Physics USSR 1: $267-279$

Sainsaulieu L (1995a) Finite volume approximation of two phase fluid flow based on approximate Roe type Riemann solver. J. Comp. Physics 121: 1-28

Sainsaulieu L (1995b) Contribution à la modélisation mathématique et numérique des écoulements diphasiques constitués d'un nuage de particules dans un écoulement de gaz. Thèse d'habilitation, Université Paris VI, Paris, France, January 20

Seguin N (2000) Comparaison numérique de schémas Volumes Finis pour les équations d'Euler en gaz parfaits et reéls. EDF report HI-81/00/010/A, Electricité de France, Chatou, France

Shih T, Lumley JL (1985) Modeling of pressure corelation terms in Reynolds stress and scalar flux equations. Cornell University report FDA-85-3

Shih T, Shabbir A, Lumley JL (1994) Realisability in second moment turbulence closures revisited. NASA Technical Memorandum 106469

Smoller J (1983) Shock waves and reaction diffusion equations. Springer Verlag, New York

Speziale CG (1979) Invariance of turbulent closure models. Physics of Fluids A 22(6): 1033-1037

Toro EF (1997) Riemann solvers and numerical methods for fluid dynamics. Springer Verlag

Toro EF, Spruce M, Speares W (1994) Restoration of the contact surface in the HLL-Riemann solver. Shock Waves 4: $25-34$

Uhlmann M (1997) Etude de modèles de fermeture au second ordre et contribution à la résolution numérique des écoulements turbulents compressibles. PhD thesis, Ecole Centrale de Lyon, Lyon, France, April 17

Vandromme D, Ha Minh H (1986) About the coupling of turbulence closure models with averaged Navier-Stokes equations. J. Comp. Physics 65(2): 386-409 